Pacific

Journal of

Mathematics

\title{
COMPLEMENTS ON DISCONNECTED REDUCTIVE GROUPS
}

FRANÇOIS Digne AND JeAn Michel

Volume 279 No. 1-2

December 2015 


\title{
COMPLEMENTS ON DISCONNECTED REDUCTIVE GROUPS
}

\author{
FRAnÇOIS Digne AND JEAN Michel \\ Dedicated to the memory of Robert Steinberg
}

\begin{abstract}
We present several results on disconnected reductive groups, in particular, on the characteristic-zero representation theory of finite groups of Lie type coming from disconnected reductive groups in positive characteristic. We generalize slightly the setting of our 1994 paper on that subject and show how most of our earlier results extend to the new situation. In particular, we give a classification of quasi-semisimple conjugacy classes over an arbitrary algebraically closed field, and over finite fields; we generalize a formula of Steinberg on the number of unipotent classes to disconnected groups and a formula for the tensor product of the Steinberg character with a Lusztig induced character.
\end{abstract}

\section{Introduction}

Let $\boldsymbol{G}$ be a (possibly disconnected) linear algebraic group over an algebraically closed field. We assume that the connected component $\boldsymbol{G}^{0}$ is reductive, and then call $\boldsymbol{G}$ a (possibly disconnected) reductive group. This situation was studied by Steinberg [1968] where he introduced the notion of quasi-semisimple elements.

Assume now that $\boldsymbol{G}$ is over an algebraic closure $\overline{\mathbb{F}}_{q}$ of the finite field $\mathbb{F}_{q}$, defined over $\mathbb{F}_{q}$ with corresponding Frobenius endomorphism $F$. Let $G^{1}$ be an $F$-stable connected component of $\boldsymbol{G}$. We want to study $\left(\boldsymbol{G}^{0}\right)^{F}$-class functions on $\left(\boldsymbol{G}^{1}\right)^{F}$; if $\boldsymbol{G}^{1}$ generates $\boldsymbol{G}$, they coincide with $\boldsymbol{G}^{F}$-class functions on $\left(\boldsymbol{G}^{1}\right)^{F}$.

This setting, adopted here, is also taken up by Lusztig in his series of papers on disconnected groups [Lusztig 2003; 2004a; 2004b; 2004c; 2004d; 2004e; 2005; 2006b; 2006a; 2009] and is slightly more general than the setting of our paper "Groupes réductifs non connexes", which we will refer to as [DM 1994], where we assumed that $\boldsymbol{G}^{1}$ contains an $F$-stable quasicentral element. A detailed comparison of both situations is done in the next section.

As the title says, this paper contains a series of complements to [DM 1994] which are mostly straightforward developments that various people have asked us about and that, except when mentioned otherwise (see the introductions to Sections 4 and

MSC2010: primary 20G15; secondary 20G40, 20C33, $20 \mathrm{G} 05$.

Keywords: nonconnected reductive groups. 
8) have not appeared in the literature, as far as we know; we thank in particular Olivier Brunat, Gerhard Hiss, Cheryl Praeger and Karine Sorlin for asking these questions.

In Section 2 we show how quite a few results of [DM 1994] are still valid in our more general setting.

In Section 3 we take a "global" viewpoint to give a formula for the scalar product of two Deligne-Lusztig characters on the whole of $\boldsymbol{G}^{F}$.

In Section 4 we show how to extend to disconnected groups the formula of Steinberg $[1968,15.1]$ counting unipotent elements.

In Section 5 we extend the theorem that tensoring Lusztig induction with the Steinberg character gives ordinary induction.

In Section 6 we give a formula for the characteristic function of a quasi-semisimple class, extending the case of a quasicentral class which was treated in [DM 1994].

In Section 7 we show how to classify quasi-semisimple conjugacy classes, first for a (possibly disconnected) reductive group over an arbitrary algebraically closed field, and then over $\mathbb{F}_{q}$.

Finally, in Section 8 we extend to our setting previous results on Shintani descent.

We thank Gunter Malle for a careful reading of the manuscript.

\section{Preliminaries}

In this paper, we consider a (possibly disconnected) algebraic group $\boldsymbol{G}$ over $\overline{\mathbb{F}}_{q}$ (except at the beginning of Section 7 where we accept an arbitrary algebraically closed field), defined over $\mathbb{F}_{q}$ with corresponding Frobenius endomorphism $F$. If $\boldsymbol{G}^{1}$ is an $F$-stable component of $\boldsymbol{G}$, we define the class functions on $\left(\boldsymbol{G}^{1}\right)^{F}$ to be the complex-valued functions invariant under $\left(\boldsymbol{G}^{0}\right)^{F}$-conjugacy (or equivalently under $\left(\boldsymbol{G}^{1}\right)^{F}$-conjugacy). Note that if $\boldsymbol{G}^{1}$ does not generate $\boldsymbol{G}$, there may be less functions invariant by $\boldsymbol{G}^{F}$-conjugacy than by $\left(\boldsymbol{G}^{1}\right)^{F}$-conjugacy; but the propositions we prove will apply in particular to the $\boldsymbol{G}^{F}$-invariant functions so we do not lose any generality. The class functions on $\left(\boldsymbol{G}^{1}\right)^{F}$ are provided with the scalar product

$$
\langle f, g\rangle_{\left(\boldsymbol{G}^{1}\right)^{F}}=\left|\left(\boldsymbol{G}^{1}\right)^{F}\right|^{-1} \sum_{h \in\left(\boldsymbol{G}^{1}\right)^{F}} f(h) \overline{g(h)} .
$$

We call $\boldsymbol{G}$ reductive when $\boldsymbol{G}^{0}$ is reductive.

When $\boldsymbol{G}$ is reductive, following [Steinberg 1968], we call an element quasi-semisimple if it normalizes a pair $\boldsymbol{T}^{0} \subset \boldsymbol{B}^{0}$ of a maximal torus of $\boldsymbol{G}^{0}$ and a Borel subgroup of $\boldsymbol{G}^{0}$. Following [DM 1994, définition-théorème 1.15], we call a quasi-semisimple element $\sigma$ quasicentral if it has maximal dimension of the centralizer $C_{\boldsymbol{G}^{0}}(\sigma)$ (that we will also denote by $\boldsymbol{G}^{0 \sigma}$ ) amongst all quasi-semisimple elements of $\boldsymbol{G}^{0} \cdot \sigma$. 
In the sequel, we fix a reductive group $\boldsymbol{G}$ and (except in the next section where we take a "global" viewpoint) an $F$-stable connected component $\boldsymbol{G}^{1}$ of $\boldsymbol{G}$. In most of [DM 1994] we assumed that $\left(\boldsymbol{G}^{1}\right)^{F}$ contained a quasicentral element. Here we do not assume this. Note however that by [DM 1994, proposition 1.34], $\boldsymbol{G}^{1}$ contains an element $\sigma$ which induces an $F$-stable quasicentral automorphism of $\boldsymbol{G}^{0}$. Such an element will be enough for our purpose, and we fix one from now on.

By [DM 1994, proposition 1.35], if $H^{1}\left(F, Z G^{0}\right)=1$ then $\left(G^{1}\right)^{F}$ contains quasicentral elements. Here is an example where $\left(G^{1}\right)^{F}$ does not contain quasicentral elements.

Example 2.1. Take $s=\left(\begin{array}{ll}\xi & 0 \\ 0 & 1\end{array}\right)$, where $\xi$ is a generator of $\mathbb{F}_{q}^{\times}$, take $\boldsymbol{G}^{0}=\mathrm{SL}_{2}$ and let $\boldsymbol{G}=\left\langle\boldsymbol{G}^{0}, s>\subset \mathrm{GL}_{2}\right.$ endowed with the standard Frobenius endomorphism on $\mathrm{GL}_{2}$, so that $s$ is $F$-stable and $\boldsymbol{G}^{F}=\mathrm{GL}_{2}\left(\mathbb{F}_{q}\right)$. We take $\boldsymbol{G}^{1}=\boldsymbol{G}^{0} \cdot s$. Here quasicentral elements are central and coincide with $\boldsymbol{G}^{0} \cdot s \cap Z \boldsymbol{G}$, which is nonempty since if $\eta \in \mathbb{F}_{q^{2}}$ is a square root of $\xi$ then $\left(\begin{array}{ll}\eta & 0 \\ 0 & \eta\end{array}\right) \in \boldsymbol{G}^{0} \cdot s \cap Z \boldsymbol{G}$; but $\boldsymbol{G}^{0} \cdot s$ does not meet $(Z \boldsymbol{G})^{F}$.

In the above example $\boldsymbol{G}^{1} / \boldsymbol{G}^{0}$ is a semisimple element of $\boldsymbol{G} / \boldsymbol{G}^{0}$. No such example exists when $\boldsymbol{G}^{1} / \boldsymbol{G}^{0}$ is unipotent:

Lemma 2.2. Let $\boldsymbol{G}^{1}$ be an $F$-stable connected component of $\boldsymbol{G}$ such that $\boldsymbol{G}^{1} / \boldsymbol{G}^{0}$ is a unipotent element of $\boldsymbol{G} / \boldsymbol{G}^{0}$. Then $\left(\boldsymbol{G}^{1}\right)^{F}$ contains unipotent quasicentral elements.

Proof. Let $\boldsymbol{T}^{0} \subset \boldsymbol{B}^{0}$ be a pair of an $F$-stable maximal torus of $\boldsymbol{G}^{0}$ and an $F$-stable Borel subgroup of $\boldsymbol{G}^{0}$. Then $N_{\boldsymbol{G}^{F}}\left(\boldsymbol{T}^{0} \subset \boldsymbol{B}^{0}\right)$ meets $\left(\boldsymbol{G}^{1}\right)^{F}$, since any two $F$-stable pairs $\boldsymbol{T}^{0} \subset \boldsymbol{B}^{0}$ are $\left(\boldsymbol{G}^{0}\right)^{F}$-conjugate. Let $s u$ be the Jordan decomposition of an element of $N_{\left(\boldsymbol{G}^{1}\right)^{F}}\left(\boldsymbol{T}^{0} \subset \boldsymbol{B}^{0}\right)$. Then $s \in \boldsymbol{G}^{0}$ since $\boldsymbol{G}^{1} / \boldsymbol{G}^{0}$ is unipotent, and $u$ is $F$-stable, unipotent and still in $N_{\left(\boldsymbol{G}^{1}\right)^{F}}\left(\boldsymbol{T}^{0} \subset \boldsymbol{B}^{0}\right)$ thus quasi-semisimple, and so is quasicentral by [DM 1994, corollaire 1.33].

Note, however, that there may exist a unipotent quasicentral element $\sigma$ which is rational as an automorphism but such that there is no rational element inducing the same automorphism.

Example 2.3. We give an example in $\boldsymbol{G}=\mathrm{SL}_{5} \rtimes<\sigma^{\prime}>$, where $\boldsymbol{G}^{0}=\mathrm{SL}_{5}$ has the standard rational structure over a finite field $\mathbb{F}_{q}$ of characteristic 2 with $q \equiv 1 \bmod 5$ and $\sigma^{\prime}$ is the automorphism of $\boldsymbol{G}^{0}$ given by $g \mapsto J^{t} g^{-1} J$ where $J$ is the antidiagonal matrix with all nonzero entries equal to 1 , so that $\sigma^{\prime}$ stabilizes the pair $\boldsymbol{T}^{0} \subset \boldsymbol{B}^{0}$, where $\boldsymbol{T}^{0}$ is the maximal torus of diagonal matrices and $\boldsymbol{B}^{0}$ is the Borel subgroup of upper triangular matrices; hence $\sigma^{\prime}$ is quasi-semisimple. Let $t$ be the diagonal matrix with entries $\left(a, a, a^{-4}, a, a\right)$, where $a^{q-1}$ is a nontrivial 5-th root of unity $\zeta \in \mathbb{F}_{q}$. We claim that $\sigma=t \sigma^{\prime}$ is as announced: it is still quasi-semisimple; we have $\sigma^{2}=t \sigma^{\prime}(t)=t t^{-1}=1$ so that $\sigma$ is unipotent; we have ${ }^{F} \sigma={ }^{F} t t^{-1} \sigma=\zeta \sigma$, so that 
$\sigma$ is rational as an automorphism but not rational. Moreover a rational element inducing the same automorphism must be of the form $z \sigma$ with $z$ central in $\boldsymbol{G}^{0}$ and $z \cdot{ }^{F} z^{-1}=\zeta \mathrm{Id}$; but the center $Z \boldsymbol{G}^{0}$ is generated by $\zeta \mathrm{Id}$ and for any $z=\zeta^{k} \operatorname{Id} \in Z \boldsymbol{G}^{0}$, we have $z \cdot{ }^{F} z^{-1}=\zeta^{k(q-1)} \mathrm{Id}=\mathrm{Id} \neq \zeta$ Id.

As in [DM 1994] we call a Levi of $\boldsymbol{G}$ a subgroup $\boldsymbol{L}$ of the form $N_{\boldsymbol{G}}\left(\boldsymbol{L}^{0} \subset \boldsymbol{P}^{0}\right)$ where $\boldsymbol{L}^{0}$ is a Levi subgroup of the parabolic subgroup $\boldsymbol{P}^{0}$ of $\boldsymbol{G}^{0}$. A particular case is a "torus" $N_{\boldsymbol{G}}\left(\boldsymbol{T}^{0}, \boldsymbol{B}^{0}\right)$ where $\boldsymbol{T}^{0} \subset \boldsymbol{B}^{0}$ is a pair of a maximal torus of $\boldsymbol{G}^{0}$ and a Borel subgroup of $\boldsymbol{G}^{0}$; note that a "torus" meets all connected components of $\boldsymbol{G}$, while (contrary to what is stated erroneously after [DM 1994, définition 1.4]) this may not be the case for a Levi.

We define a Levi of $\boldsymbol{G}^{1}$ to be a set of the form $\boldsymbol{L}^{1}=\boldsymbol{L} \cap \boldsymbol{G}^{1}$, where $\boldsymbol{L}$ is a Levi of $\boldsymbol{G}$ and the intersection is nonempty; note that if $\boldsymbol{G}^{1}$ does not generate $\boldsymbol{G}$, there may exist several Levis of $\boldsymbol{G}$ which have same intersection with $\boldsymbol{G}^{1}$. Nevertheless $\boldsymbol{L}^{1}$ determines $\boldsymbol{L}^{0}$ as the identity component of $\left\langle\boldsymbol{L}^{1}\right\rangle$.

We assume now that $\boldsymbol{L}^{1}$ is an $F$-stable Levi of $\boldsymbol{G}^{1}$ of the form $N_{\boldsymbol{G}^{1}}\left(\boldsymbol{L}^{0} \subset \boldsymbol{P}^{0}\right)$. If $\boldsymbol{U}$ is the unipotent radical of $\boldsymbol{P}^{0}$, we define $\boldsymbol{Y}_{\boldsymbol{U}}^{0}=\left\{x \in \boldsymbol{G}^{0} \mid x^{-1} \cdot{ }^{F} x \in \boldsymbol{U}\right\}$ on which $(g, l) \in \boldsymbol{G}^{F} \times \boldsymbol{L}^{F}$ such that $g l \in \boldsymbol{G}^{0}$ acts by $x \mapsto g x l$, where $\boldsymbol{L}=N_{\boldsymbol{G}}\left(\boldsymbol{L}^{0}, \boldsymbol{P}^{0}\right)$. Along the same lines as [DM 1994, proposition 2.10] we define the following:

Definition 2.4. Let $\boldsymbol{L}^{1}$ be an $F$-stable Levi of $\boldsymbol{G}^{1}$ of the form $N_{\boldsymbol{G}^{1}}\left(\boldsymbol{L}^{0} \subset \boldsymbol{P}^{0}\right)$ and let $\boldsymbol{U}$ be the unipotent radical of $\boldsymbol{P}^{0}$. For $\lambda$ a class function on $\left(\boldsymbol{L}^{1}\right)^{F}$ and $g \in\left(\boldsymbol{G}^{1}\right)^{F}$, we set

$$
R_{\boldsymbol{L}^{1}}^{\boldsymbol{G}^{1}}(\lambda)(g)=\left|\left(\boldsymbol{L}^{1}\right)^{F}\right|^{-1} \sum_{l \in\left(\boldsymbol{L}^{1}\right)^{F}} \lambda(l) \operatorname{Trace}\left(\left(g, l^{-1}\right) \mid H_{c}^{*}\left(\boldsymbol{Y}_{\boldsymbol{U}}^{0}\right)\right),
$$

and for $\gamma$ a class function on $\left(\boldsymbol{G}^{1}\right)^{F}$ and $l \in\left(\boldsymbol{L}^{1}\right)^{F}$, we set

$$
{ }^{*} R_{\boldsymbol{L}^{1}}^{\boldsymbol{G}^{1}}(\gamma)(l)=\left|\left(\boldsymbol{G}^{1}\right)^{F}\right|^{-1} \sum_{g \in\left(\boldsymbol{G}^{1}\right)^{F}} \gamma(g) \operatorname{Trace}\left(\left(g^{-1}, l\right) \mid H_{c}^{*}\left(\boldsymbol{Y}_{\boldsymbol{U}}^{0}\right)\right) .
$$

In the above, $H_{c}^{*}$ denotes the $\ell$-adic cohomology with compact support, where we have chosen once and for all a prime number $\ell \neq p$. In order to consider the virtual character $\operatorname{Trace}\left(x \mid H_{c}^{*}(\boldsymbol{X})\right)=\sum_{i}(-1)^{i} \operatorname{Trace}\left(x \mid H_{c}^{i}\left(\boldsymbol{X}, \overline{\mathbb{Q}}_{\ell}\right)\right)$ as a complex character we chose once and for all an embedding $\overline{\mathbb{Q}}_{\ell} \hookrightarrow \mathbb{C}$.

Writing $R_{L^{1}}^{G^{1}}$ and ${ }^{*} R_{L^{1}}^{G^{1}}$ is an abuse of notation: the definition needs the choice of a $\boldsymbol{P}^{0}$ such that $\boldsymbol{L}^{1}=N_{\boldsymbol{G}^{1}}\left(\boldsymbol{L}^{0} \subset \boldsymbol{P}^{0}\right)$. Our subsequent statements will use an implicit choice. Under certain assumptions, we will prove a Mackey formula (Theorem 2.6) which when true implies that $R_{\boldsymbol{L}^{1}}^{G^{1}}$ and ${ }^{*} R_{\boldsymbol{L}^{1}}^{G^{1}}$ are independent of the choice of $\boldsymbol{P}^{0}$.

By the same arguments as for [DM 1994, proposition 2.10] (using that $\left(\boldsymbol{L}^{1}\right)^{F}$ is nonempty and [DM 1994, proposition 2.3]) Definition 2.4 agrees with the restriction to $\left(\boldsymbol{G}^{1}\right)^{F}$ and $\left(\boldsymbol{L}^{1}\right)^{F}$ of [DM 1994, définition 2.2]. 
The two maps $R_{L^{1}}^{G^{1}}$ and ${ }^{*} R_{L^{1}}^{G^{1}}$ are adjoint with respect to the scalar products on $\left(\boldsymbol{G}^{1}\right)^{F}$ and $\left(\boldsymbol{L}^{1}\right)^{F}$.

We note the following variation on [DM 1994, proposition 2.6] where, for $u$ (resp. $v$ ) a unipotent element of $\boldsymbol{G}($ resp. $\boldsymbol{L})$, we set

$$
Q_{\boldsymbol{L}^{0}}^{\boldsymbol{G}^{0}}(u, v)= \begin{cases}\operatorname{Trace}\left((u, v) \mid H_{c}^{*}\left(\boldsymbol{Y}_{\boldsymbol{U}}^{0}\right)\right) & \text { if } u v \in \boldsymbol{G}^{0}, \\ 0 & \text { otherwise. }\end{cases}
$$

Proposition 2.5. Let su be the Jordan decomposition of an element of $\left(\boldsymbol{G}^{1}\right)^{F}$ and $\lambda$ a class function on $\left(\boldsymbol{L}^{1}\right)^{F}$.

(i) If $s$ is central in $\boldsymbol{G}$, we have

$$
\left(R_{\boldsymbol{L}^{1}}^{\boldsymbol{G}^{1}} \lambda\right)(s u)=\left|\left(\boldsymbol{L}^{0}\right)^{F}\right|^{-1} \sum_{v \in\left(\boldsymbol{L}^{0} \cdot u\right)_{\mathrm{unip}}^{F}} Q_{\boldsymbol{L}^{0}}^{\boldsymbol{G}^{0}}\left(u, v^{-1}\right) \lambda(s v) .
$$

(ii) In general,

$$
\left(R_{\boldsymbol{L}^{1}}^{\boldsymbol{G}^{1}} \lambda\right)(s u)=\sum_{\left\{\left.h \in\left(\boldsymbol{G}^{0}\right)^{F}\right|^{h} \boldsymbol{L} \ni s\right\}} \frac{\left|{ }^{h} \boldsymbol{L}^{0} \cap C_{\boldsymbol{G}}(s)^{0 F}\right|}{\left|\left(\boldsymbol{L}^{0}\right)^{F}\right|\left|C_{\boldsymbol{G}}(s)^{0 F}\right|} R_{h}^{C_{\boldsymbol{G}}(s)^{0} \cdot C_{\boldsymbol{G}}(s)^{0} \cdot s u}\left({ }^{h} \lambda\right)(s u) .
$$

(iii) If t $v$ is the Jordan decomposition of an element of $\left(\boldsymbol{L}^{1}\right)^{F}$ and $\gamma$ a class function on $\left(\boldsymbol{G}^{1}\right)^{F}$, we have

$$
\left({ }^{*} R_{\boldsymbol{L}^{1}}^{\boldsymbol{G}^{1}} \gamma\right)(t v)=\left|\left(\boldsymbol{G}^{t 0}\right)^{F}\right|^{-1} \sum_{\left.u \in\left(\boldsymbol{G}^{t 0} \cdot v\right)\right)_{\text {unip }}^{F}} Q_{\boldsymbol{L}^{t 0}}^{\boldsymbol{G}^{t 0}}\left(u, v^{-1}\right) \gamma(t u) .
$$

In the above we abused notation to write ${ }^{h} \boldsymbol{L} \ni s$ for $\left\langle\boldsymbol{L}^{1}\right\rangle \ni{ }^{h^{-1}} s$.

Proof. Part (i) results from [DM 1994, proposition 2.6(i)] using the same arguments as the proof of [DM 1994, propsition 2.10]; we then get (ii) by plugging (i) into [DM 1994, proposition 2.6(i)].

In our setting the Mackey formula [DM 1994, définition 3.1] is still valid in the cases where we proved it: théorèmes 3.2 and 4.5 in [DM 1994]. Before stating it we remark that [DM 1994, proposition 1.40] remains true without the assumption that $\left(\boldsymbol{G}^{1}\right)^{F}$ contains quasicentral elements; we need only replace $\left(\boldsymbol{G}^{0}\right)^{F} . \sigma$ with $\left(\boldsymbol{G}^{1}\right)^{F}$ in the proof. Thus any $F$-stable Levi of $\boldsymbol{G}^{1}$ is $\left(\boldsymbol{G}^{0}\right)^{F}$-conjugate to a Levi containing $\sigma$. This explains why we only state the Mackey formula in the case of Levis containing $\sigma$.

Theorem 2.6. If $\boldsymbol{L}^{1}$ and $\boldsymbol{M}^{1}$ are two $F$-stable Levis of $\boldsymbol{G}^{1}$ containing $\sigma$ then under one of the following assumptions:

- $\boldsymbol{L}^{0}\left(\right.$ resp. $\left.\boldsymbol{M}^{0}\right)$ is a Levi subgroup of an $F$-stable parabolic subgroup normalized by $\boldsymbol{L}^{1}$ (resp. $\left.\boldsymbol{M}^{1}\right)$,

- one of $\boldsymbol{L}^{1}$ and $\boldsymbol{M}^{1}$ is a "torus", 
we have

$$
{ }^{*} R_{\boldsymbol{L}^{1}}^{\boldsymbol{G}^{1}} R_{\boldsymbol{M}^{1}}^{\boldsymbol{G}^{1}}=\sum_{x \in\left[\boldsymbol{L}^{\sigma 0^{F}} \backslash \mathcal{S}_{\boldsymbol{G}^{\sigma 0}}\left(\boldsymbol{L}^{\sigma 0}, \boldsymbol{M}^{\sigma 0}\right)^{F} / \boldsymbol{M}^{\sigma 0^{F}}\right]} R_{\left(\boldsymbol{L}^{1} \cap{ }^{x} \boldsymbol{M}^{1}\right)}^{\boldsymbol{L}^{1}}{ }^{*} R_{\left(\boldsymbol{L}^{1} \cap^{x} \boldsymbol{M}^{1}\right)}^{\boldsymbol{M}^{1}} \text { ad } x,
$$

where $\mathcal{S}_{\boldsymbol{G}^{\sigma 0}}\left(\boldsymbol{L}^{\sigma 0}, \boldsymbol{M}^{\sigma 0}\right)$ is the set of elements $x \in \boldsymbol{G}^{\sigma 0}$ such that $\boldsymbol{L}^{\sigma 0} \cap^{x} \boldsymbol{M}^{\sigma 0}$ contains a maximal torus of $\boldsymbol{G}^{\sigma 0}$.

Proof. We first prove the theorem in the case of $F$-stable parabolic subgroups $\boldsymbol{P}^{0}=\boldsymbol{L}^{0} \ltimes \boldsymbol{U}$ and $\boldsymbol{Q}^{0}=\boldsymbol{M}^{0} \ltimes \boldsymbol{V}$ following the proof of [DM 1994, théorème 3.2]. The difference is that the variety we consider here is the intersection with $\boldsymbol{G}^{0}$ of the variety considered in [loc. cit.]. Here, the left-hand side of the Mackey formula is given by $\overline{\mathbb{Q}}_{\ell}\left[\left(\boldsymbol{U}^{F} \backslash\left(\boldsymbol{G}^{0}\right)^{F} / \boldsymbol{V}^{F}\right)^{\sigma}\right]$ instead of $\overline{\mathbb{Q}}_{\ell}\left[\left(\boldsymbol{U}^{F} \backslash\left(\boldsymbol{G}^{0}\right)^{F} .<\sigma>/ \boldsymbol{V}^{F}\right)^{\sigma}\right]$. Nevertheless we can use [DM 1994, lemme 3.3], which remains valid with the same proof. As for [DM 1994, lemme 3.5], we have to replace it with the following:

Lemma 2.7. For any $x \in \mathcal{S}_{\boldsymbol{G}^{\sigma 0}}\left(\boldsymbol{L}^{\sigma 0}, \boldsymbol{M}^{\sigma 0}\right)^{F}$, the map

$$
\left(l\left(\boldsymbol{L}^{0} \cap{ }^{x} \boldsymbol{V}^{F}\right),\left({ }^{x} \boldsymbol{M}^{0} \cap \boldsymbol{U}^{F}\right) \cdot{ }^{x} m\right) \mapsto \boldsymbol{U}^{F} \operatorname{lxm} \boldsymbol{V}^{F}
$$

is an isomorphism from $\left(\boldsymbol{L}^{0}\right)^{F} /\left(\boldsymbol{L}^{0} \cap{ }^{x} \boldsymbol{V}^{F}\right) \times\left(\boldsymbol{L}^{0} \cap^{x} \boldsymbol{M}^{0}\right)^{F}\left({ }^{x} \boldsymbol{M}^{0} \cap \boldsymbol{U}^{F}\right) \backslash^{x}\left(\boldsymbol{M}^{0}\right)^{F}$ to $\boldsymbol{U}^{F} \backslash\left(\boldsymbol{P}^{0}\right)^{F} x\left(\boldsymbol{Q}^{0}\right)^{F} / \boldsymbol{V}^{F}$, compatible with the following action of $\left(\boldsymbol{L}^{1}\right)^{F} \times\left(\left(\boldsymbol{M}^{1}\right)^{F}\right)^{-1}$ : $\left(\lambda, \mu^{-1}\right) \in\left(\boldsymbol{L}^{1}\right)^{F} \times\left(\left(\boldsymbol{M}^{1}\right)^{F}\right)^{-1}$ acts by mapping $\left(l\left(\boldsymbol{L}^{0} \cap{ }^{x} \boldsymbol{V}^{F}\right),\left({ }^{x} \boldsymbol{M}^{0} \cap \boldsymbol{U}^{F}\right) \cdot{ }^{x} m\right)$ to the class of $\left(\lambda l v^{-1}\left(\boldsymbol{L}^{0} \cap{ }^{x} \boldsymbol{V}^{F}\right),\left({ }^{x} \boldsymbol{M}^{0} \cap \boldsymbol{U}^{F}\right) \cdot v^{x} m \mu^{-1}\right)$ with $v \in\left(\boldsymbol{L}^{1}\right)^{F} \cap{ }^{x}\left(\boldsymbol{M}^{1}\right)^{F}$ (independent of $v$ ).

Proof. The isomorphism of the lemma involves only connected groups and is a known result (see, e.g., [Digne and Michel 1991, 5.7]). The compatibility with the actions is straightforward.

This allows us to complete the proof in the first case.

We now prove the second case following Section 4 of [DM 1994]. We first notice that the statement and proof of lemme 4.1 in [DM 1994] don't use the element $\sigma$ but only its action. In lemmes 4.2, 4.3 and 4.4 there is no $\sigma$ involved but only the action of the groups $\boldsymbol{L}^{F}$ and $\boldsymbol{M}^{F}$ on the pieces of a variety depending only on $\boldsymbol{L}, \boldsymbol{M}$ and the associated parabolics. This gives the second case.

We now rephrase [DM 1994, proposition 4.8] and [DM 1994, proposition 4.11] in our setting, specializing the Mackey formula to the case of two "tori". Let $\mathcal{T}_{1}$ be the set of "tori" of $\boldsymbol{G}^{1}$; if $\boldsymbol{T}^{1}=N_{\boldsymbol{G}^{1}}\left(\boldsymbol{T}^{0}, \boldsymbol{B}^{0}\right) \in \mathcal{T}_{1}^{F}$ then $\boldsymbol{T}^{0}$ is $F$-stable. We define $\operatorname{Irr}\left(\left(\boldsymbol{T}^{1}\right)^{F}\right)$ as the set of restrictions to $\left(\boldsymbol{T}^{1}\right)^{F}$ of extensions to $\left\langle\left(\boldsymbol{T}^{1}\right)^{F}>\right.$ of elements of $\operatorname{Irr}\left(\left(\boldsymbol{T}^{0}\right)^{F}\right)$.

Proposition 2.8. If $\boldsymbol{T}^{1}, \boldsymbol{T}^{\prime 1} \in \mathcal{T}_{1}^{F}$ and $\theta \in \operatorname{Irr}\left(\left(\boldsymbol{T}^{1}\right)^{F}\right), \theta^{\prime} \in \operatorname{Irr}\left(\left(\boldsymbol{T}^{\prime 1}\right)^{F}\right)$ then

$$
\left\langle R_{\boldsymbol{T}^{1}}^{\boldsymbol{G}^{1}}(\theta), R_{\boldsymbol{T}^{\prime 1}}^{\boldsymbol{G}^{1}}\left(\theta^{\prime}\right)\right\rangle_{\left(\boldsymbol{G}^{1}\right)^{F}}=0
$$


unless $\left(\boldsymbol{T}^{1}, \theta\right)$ and $\left(\boldsymbol{T}^{\prime 1}, \theta^{\prime}\right)$ are $\left(\boldsymbol{G}^{0}\right)^{F}$-conjugate.

Additionally,

(i) iffor some $n \in N_{\left(\boldsymbol{G}^{0}\right)^{F}}\left(\boldsymbol{T}^{1}\right)$ and $\zeta \neq 1$ we have ${ }^{n} \theta=\zeta \theta$ then $R_{\boldsymbol{T}^{1}}^{\boldsymbol{G}^{1}}(\theta)=0$;

(ii) otherwise $\left\langle R_{\boldsymbol{T}^{1}}^{\boldsymbol{G}^{1}}(\theta), R_{\boldsymbol{T}^{1}}^{\boldsymbol{G}^{1}}(\theta)\right\rangle_{\left(\boldsymbol{G}^{1}\right)^{F}}=\left|\left\{n \in N_{\left(\boldsymbol{G}^{0}\right)^{F}}\left(\boldsymbol{T}^{1}\right) \mid{ }^{n} \theta=\theta\right\}\right| /\left|\left(\boldsymbol{T}^{1}\right)^{F}\right|$.

If $\boldsymbol{T}^{1}=\boldsymbol{T}^{\prime 1}$, the above can be written as

$$
\left\langle R_{\boldsymbol{T}^{1}}^{\boldsymbol{G}^{1}}(\theta), R_{\boldsymbol{T}^{1}}^{\boldsymbol{G}^{1}}\left(\theta^{\prime}\right)\right\rangle_{\left(\boldsymbol{G}^{1}\right)^{F}}=\left\langle\operatorname{Ind}_{\left(\boldsymbol{T}^{1}\right)^{F}}^{N_{\boldsymbol{G}^{1}}\left(\boldsymbol{T}^{0}\right)^{F}} \theta, \operatorname{Ind}_{\left(\boldsymbol{T}^{1}\right)^{F}}^{N_{\boldsymbol{G}^{1}}\left(\boldsymbol{T}^{0}\right)^{F}} \theta^{\prime}\right\rangle_{N_{\boldsymbol{G}^{1}}\left(\boldsymbol{T}^{0}\right)^{F}},
$$

where when $A^{1} \subset B^{1}$ are cosets of finite groups $A^{0} \subset B^{0}$ and $\chi$ is an $A^{0}$-class function on $A^{1}$ for $x \in B^{1}$, we set $\operatorname{Ind}_{A^{1}}^{B^{1}} \chi(x)=\left|A^{0}\right|^{-1} \sum_{\left\{y \in B^{0} \mid y_{x} \in A^{1}\right\}} \chi\left({ }^{y} x\right)$.

Proof. As noticed above Theorem 2.6, we may assume that $\boldsymbol{T}^{1}$ and $\boldsymbol{T}^{\prime 1}$ contain $\sigma$. By [DM 1994, proposition 1.39], if $\boldsymbol{T}^{1}$ and $\boldsymbol{T}^{\prime 1}$ contain $\sigma$, they are $\left(\boldsymbol{G}^{0}\right)^{F}$-conjugate if and only if they are conjugate under $\boldsymbol{G}^{\sigma 0^{F}}$. The Mackey formula shows then that the scalar product vanishes when $\boldsymbol{T}^{1}$ and $\boldsymbol{T}^{\prime 1}$ are not $\left(\boldsymbol{G}^{0}\right)^{F}$-conjugate.

Otherwise we may assume $\boldsymbol{T}^{1}=\boldsymbol{T}^{\prime 1}$ and the Mackey formula gives

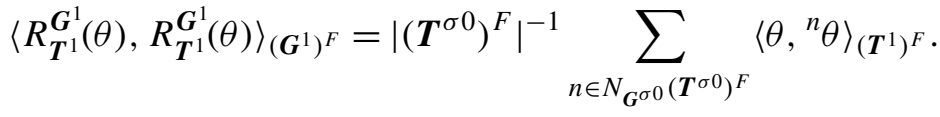

The term $\left\langle\theta,{ }^{n} \theta\right\rangle_{\left(\boldsymbol{T}^{1}\right)^{F}}$ is 0 unless ${ }^{n} \theta=\zeta_{n} \theta$ for some constant $\zeta_{n}$ and, in this last case, $\left\langle\theta,{ }^{n} \theta\right\rangle_{\left(\boldsymbol{T}^{1}\right)^{F}}=\bar{\zeta}_{n}$. If ${ }^{n^{\prime}} \theta=\zeta_{n^{\prime}} \theta$ then ${ }^{n n^{\prime}} \theta=\zeta_{n^{\prime}}{ }^{n} \theta=\zeta_{n^{\prime}} \zeta_{n} \theta$, and thus the $\zeta_{n}$ form a group; if this group is not trivial, that is, some $\zeta_{n}$ is not equal to 1 , we have

$$
\left\langle R_{\boldsymbol{T}^{1}}^{\boldsymbol{G}^{1}}(\theta), R_{\boldsymbol{T}^{1}}^{\boldsymbol{G}^{1}}(\theta)\right\rangle_{\left(\boldsymbol{G}^{1}\right)^{F}}=0,
$$

which implies that in this case $R_{T^{1}}^{G^{1}}(\theta)=0$. This gives (i) since by [DM 1994, proposition 1.39], if $\boldsymbol{T}^{1} \ni \sigma$ then $N_{\left(\boldsymbol{G}^{0}\right)^{F}}\left(\boldsymbol{T}^{1}\right)=N_{\boldsymbol{G}^{\sigma 0}}\left(\boldsymbol{T}^{\sigma 0}\right)^{F} \cdot\left(\boldsymbol{T}^{0}\right)^{F}$, so that if there exists $n$ as in (i), there exists an $n \in N_{\boldsymbol{G}^{\sigma 0}}\left(\boldsymbol{T}^{\sigma 0}\right)^{F}$ with same action on $\theta$ since $\left(\boldsymbol{T}^{0}\right)^{F}$ has trivial action on $\theta$.

In case (ii), for each nonzero term we have ${ }^{n} \theta=\theta$ and we have to check that the value $\left|\left(\left(\boldsymbol{T}^{\sigma}\right)^{0}\right)^{F}\right|^{-1}\left|\left\{\left.n \in N_{\boldsymbol{G}^{\sigma 0}}\left(\boldsymbol{T}^{\sigma 0}\right)^{F}\right|^{n} \theta=\theta\right\}\right|$ given by the Mackey formula is equal to the stated value. This results again from [DM 1994, proposition 1.39], written as $N_{\left(\boldsymbol{G}^{0}\right)^{F}}\left(\boldsymbol{T}^{1}\right)=N_{\boldsymbol{G}^{\sigma 0}}\left(\boldsymbol{T}^{1}\right)^{F} \cdot\left(\boldsymbol{T}^{0}\right)^{F}$, and from $N_{\boldsymbol{G}^{\sigma 0}}\left(\boldsymbol{T}^{1}\right)^{F} \cap\left(\boldsymbol{T}^{0}\right)^{F}=\left(\left(\boldsymbol{T}^{\sigma}\right)^{0}\right)^{F}$.

We now prove the final remark. By definition we have

$$
\begin{aligned}
& \left\langle\operatorname{Ind}_{\left(\boldsymbol{T}^{1}\right)^{F}}^{N_{\boldsymbol{G}^{1}}\left(\boldsymbol{T}^{0}\right)^{F}} \theta, \operatorname{Ind}_{\left(\boldsymbol{T}^{1}\right)^{F}}^{N_{\boldsymbol{G}^{1}}\left(\boldsymbol{T}^{0}\right)^{F}} \theta^{\prime}\right\rangle_{N_{\boldsymbol{G}^{1}}\left(\boldsymbol{T}^{0}\right)^{F}} \\
& \quad=\left|N_{\boldsymbol{G}^{1}}\left(\boldsymbol{T}^{0}\right)^{F}\right|^{-1}\left|\left(\boldsymbol{T}^{1}\right)^{F}\right|^{-2} \sum_{x \in N_{\boldsymbol{G}^{1}}\left(\boldsymbol{T}^{0}\right)^{F}} \sum_{\left\{n,\left.n^{\prime} \in N_{\boldsymbol{G}^{1}}\left(\boldsymbol{T}^{0}\right)^{F}\right|^{n} x, n^{\prime} x \in \boldsymbol{T}^{1}\right\}} \theta\left({ }^{n} x\right) \overline{\theta\left(n^{\prime} x\right)} .
\end{aligned}
$$

Doing the summation over $t={ }^{n} x$ and $n^{\prime \prime}=n^{\prime} n^{-1} \in N_{\boldsymbol{G}^{0}}\left(\boldsymbol{T}^{0}\right)^{F}$, we get

$$
\left|N_{\boldsymbol{G}^{1}}\left(\boldsymbol{T}^{0}\right)^{F}\right|^{-1}\left|\left(\boldsymbol{T}^{1}\right)^{F}\right|^{-2} \sum_{t \in\left(\boldsymbol{T}^{1}\right)^{F}} \sum_{n \in N_{\boldsymbol{G}^{1}}\left(\boldsymbol{T}^{0}\right)^{F}} \sum_{\left\{n^{\prime \prime} \in N_{\boldsymbol{G}^{0}}\left(\boldsymbol{T}^{0}\right)^{F} \mid n^{\prime \prime} t \in \boldsymbol{T}^{1}\right\}} \theta(t) \overline{\theta\left(n^{\prime \prime} t\right)} .
$$


The condition $n^{\prime \prime} \in N_{\boldsymbol{G}^{0}}\left(\boldsymbol{T}^{0}\right)^{F}$, together with ${ }^{n^{\prime \prime}} t \in \boldsymbol{T}^{1}$, is equivalent to $n^{\prime \prime} \in$ $N_{G^{0}}\left(\boldsymbol{T}^{1}\right)^{F}$, so that we get

$$
\left|\left(\boldsymbol{T}^{1}\right)^{F}\right|^{-1} \sum_{n^{\prime \prime} \in N_{\boldsymbol{G}^{0}}\left(\boldsymbol{T}^{1}\right)^{F}}\left\langle\theta,{ }^{n^{\prime \prime}} \theta\right\rangle_{\left(\boldsymbol{T}^{1}\right)^{F}} .
$$

As explained in the first part of the proof, the scalar product $\left\langle\theta,{ }^{n^{\prime \prime}} \theta\right\rangle_{\left(\boldsymbol{T}^{1}\right)^{F}}$ is zero unless ${ }^{n^{\prime \prime}} \theta=\zeta_{n^{\prime \prime}} \theta$ for some root of unity $\zeta_{n^{\prime \prime}}$ and arguing as in the first part of the proof, we find that the above sum is zero if there exists $n^{\prime \prime}$ such that $\zeta_{n^{\prime \prime}} \neq 1$ and is equal to $\left|\left(\boldsymbol{T}^{1}\right)^{F}\right|^{-1}\left|\left\{n \in N_{\left(\boldsymbol{G}^{0}\right)^{F}}\left(\boldsymbol{T}^{1}\right) \mid{ }^{n} \theta=\theta\right\}\right|$ otherwise.

Remark 2.9. In the context of Proposition 2.8, if $\sigma$ is $F$-stable then we may apply $\theta$ to it and for any $n \in N_{\boldsymbol{G}^{\sigma 0}}\left(\boldsymbol{T}^{\sigma 0}\right)^{F}$, we have $\theta\left({ }^{n} \sigma\right)=\theta(\sigma)$, so for any $n \in N_{\left(\boldsymbol{G}^{0}\right)^{F}}\left(\boldsymbol{T}^{1}\right)$ and $\zeta$ such that ${ }^{n} \theta=\zeta \theta$, we have $\zeta=1$. When $H^{1}\left(F, Z \boldsymbol{G}^{0}\right)=1$, we may choose $\sigma$ to be $F$-stable so that $\zeta \neq 1$ never happens.

Here is an example where $\zeta_{n}=-1$, and thus $R_{T^{1}}^{G^{1}}(\theta)=0$ : we take again the context of Example 2.1 and take $\boldsymbol{T}^{0}=\left\{\left(\begin{array}{cc}a & 0 \\ 0 & a^{-1}\end{array}\right)\right\}$ and let $\boldsymbol{T}^{1}=\boldsymbol{T}^{0} \cdot s$; let us define $\theta$ on $t s \in\left(\boldsymbol{T}^{1}\right)^{F}$ by $\theta(t s)=-\lambda(t)$, where $\lambda$ is the nontrivial order-2 character of $\left(\boldsymbol{T}^{0}\right)^{F}$ (Legendre symbol); then for any $n \in N_{\left(\boldsymbol{G}^{0}\right)^{F}}\left(\boldsymbol{T}^{1}\right) \backslash \boldsymbol{T}^{0}$, we have ${ }^{n} \theta=-\theta$.

We define uniform functions as the class functions on $\left(G^{1}\right)^{F}$ which are linear combinations of the $R_{\boldsymbol{T}^{1}}^{\boldsymbol{G}^{1}}(\theta)$ for $\theta \in \operatorname{Irr}\left(\left(\boldsymbol{T}^{1}\right)^{F}\right)$. Proposition 4.11 in [DM 1994] extends as follows to our context:

Corollary 2.10 (of Proposition 2.8). Let $p^{G^{1}}$ be the projector to uniform functions on $\left(\boldsymbol{G}^{1}\right)^{F}$. We have

$$
p^{G^{1}}=\left|\left(G^{1}\right)^{F}\right|^{-1} \sum_{T^{1} \in \mathcal{T}_{1}^{F}}\left|\left(T^{1}\right)^{F}\right| R_{T^{1}}^{G^{1}}{ }^{*} R_{T^{1}}^{G^{1}} .
$$

Proof. We need only check that for any $\theta \in \operatorname{Irr}\left(\left(\boldsymbol{T}^{1}\right)^{F}\right)$ such that $R_{\boldsymbol{T}^{1}}^{\boldsymbol{G}^{1}}(\theta) \neq 0$ and any class function $\chi$ on $\left(\boldsymbol{G}^{1}\right)^{F}$, we have $\left\langle p^{\boldsymbol{G}^{1}} \chi, R_{\boldsymbol{T}^{1}}^{\boldsymbol{G}^{1}}(\theta)\right\rangle_{\left(\boldsymbol{G}^{1}\right)^{F}}=\left\langle\chi, R_{\boldsymbol{T}^{1}}^{\boldsymbol{G}^{1}}(\theta)\right\rangle_{\left(\boldsymbol{G}^{1}\right)^{F}}$. By Proposition 2.8, to evaluate the left-hand side we may restrict the sum to tori conjugate to $\boldsymbol{T}^{1}$, so we get

$$
\begin{aligned}
& \left\langle p^{\boldsymbol{G}^{1}} \chi, R_{\boldsymbol{T}^{1}}^{\boldsymbol{G}^{1}}(\theta)\right\rangle_{\left(\boldsymbol{G}^{1}\right)^{F}}=\left|N_{\left(\boldsymbol{G}^{0}\right)^{F}}\left(\boldsymbol{T}^{1}\right)\right|^{-1}\left|\left(\boldsymbol{T}^{1}\right)^{F}\right|\left\langle R_{\boldsymbol{T}^{1}}^{\boldsymbol{G}^{1}{ }^{*}} R_{\boldsymbol{T}^{1}}^{\boldsymbol{G}^{1}} \chi, R_{\boldsymbol{T}^{1}}^{\boldsymbol{G}^{1}}(\theta)\right\rangle_{\left(\boldsymbol{G}^{1}\right)^{F}} \\
& =\left|N_{\left(\boldsymbol{G}^{0}\right)^{F}}\left(\boldsymbol{T}^{1}\right)\right|^{-1}\left|\left(\boldsymbol{T}^{1}\right)^{F}\right|\left\langle\chi, R_{\boldsymbol{T}^{1}}^{\boldsymbol{G}^{1} \circ R^{*} \boldsymbol{T}^{1}} \boldsymbol{G}^{1} \circ R_{\boldsymbol{T}^{1}}^{\boldsymbol{G}^{1}}(\theta)\right\rangle_{\left(\boldsymbol{G}^{1}\right)^{F}} .
\end{aligned}
$$

The equality to be proved is true if $R_{T^{1}}^{G^{1}}(\theta)=0$; otherwise by Proposition 2.8, we have ${ }^{*} R_{\boldsymbol{T}^{1}}^{\boldsymbol{G}^{1}} \circ R_{\boldsymbol{T}^{1}}^{\boldsymbol{G}^{1}}(\theta)=\left|\left(\boldsymbol{T}^{1}\right)^{F}\right|^{-1} \sum_{n \in N_{\left(\boldsymbol{G}^{0}\right)^{F}}\left(\boldsymbol{T}^{1}\right)}{ }^{n} \theta$, whence in that case

$$
R_{\boldsymbol{T}^{1}}^{\boldsymbol{G}^{1}}{ }^{*} R_{\boldsymbol{T}^{1}}^{\boldsymbol{G}^{1}} \circ R_{\boldsymbol{T}^{1}}^{\boldsymbol{G}^{1}}(\theta)=\left|\left(\boldsymbol{T}^{1}\right)^{F}\right|^{-1}\left|N_{\left(\boldsymbol{G}^{0}\right)^{F}}\left(\boldsymbol{T}^{1}\right)\right| R_{\boldsymbol{T}^{1}}^{\boldsymbol{G}^{1}}(\theta)
$$

since $R_{T^{1}}^{G^{1}}\left({ }^{n} \theta\right)=R_{T^{1}}^{G^{1}}(\theta)$, and hence the result.

We now adapt the definition of duality to our setting. 
Definition 2.11. - For a connected reductive group $\boldsymbol{G}$, we define the $\mathbb{F}_{q}$-rank as the maximal dimension of a split torus, and define $\varepsilon_{\boldsymbol{G}}=(-1)^{\mathbb{F}_{q} \text {-rank of } \boldsymbol{G}}$ and $\eta_{\boldsymbol{G}}=\varepsilon_{\boldsymbol{G}} / \operatorname{rad} \boldsymbol{G}$.

- For an $F$-stable connected component $\boldsymbol{G}^{1}$ of a (possibly disconnected) reductive group, we define $\varepsilon_{\boldsymbol{G}^{1}}=\varepsilon_{\boldsymbol{G}^{\sigma 0}}$ and $\eta_{\boldsymbol{G}^{1}}=\eta_{\boldsymbol{G}^{\sigma 0}}$, where $\sigma \in \boldsymbol{G}^{1}$ induces an $F$-stable quasicentral automorphism of $\boldsymbol{G}^{0}$.

Let us see that these definitions agree with [DM 1994]: in [DM 1994, définition 3.6(i)], we define $\varepsilon_{\boldsymbol{G}^{1}}$ to be $\varepsilon_{\boldsymbol{G}^{0 \tau}}$, where $\tau$ is any quasi-semisimple element of $\boldsymbol{G}^{1}$ which induces an $F$-stable automorphism of $\boldsymbol{G}^{0}$ and lies in a "torus" of the form $N_{\boldsymbol{G}^{1}}\left(\boldsymbol{T}_{0} \subset \boldsymbol{B}_{0}\right)$, where both $\boldsymbol{T}^{0}$ and $\boldsymbol{B}^{0}$ are $F$-stable; by [DM 1994, proposition 1.36(ii)], a $\sigma$ as above is such a $\tau$.

We fix an $F$-stable pair $\left(\boldsymbol{T}_{0} \subset \boldsymbol{B}_{0}\right)$ and define duality on $\operatorname{Irr}\left(\left(\boldsymbol{G}^{1}\right)^{F}\right)$ by

$$
D_{\boldsymbol{G}^{1}}=\sum_{\boldsymbol{P}^{0} \supset \boldsymbol{B}^{0}} \eta_{\boldsymbol{L}^{1}} R_{\boldsymbol{L}^{1}}^{\boldsymbol{G}^{1}}{ }^{*} R_{\boldsymbol{L}^{1}}^{\boldsymbol{G}^{1}}
$$

where in the sum, $\boldsymbol{P}^{0}$ runs over $F$-stable parabolic subgroups containing $\boldsymbol{B}^{0}$ such that $N_{\boldsymbol{G}^{1}}\left(\boldsymbol{P}^{0}\right)$ is nonempty, and $\boldsymbol{L}^{1}$ denotes $N_{\boldsymbol{G}^{1}}\left(\boldsymbol{L}^{0} \subset \boldsymbol{P}^{0}\right)$, where $\boldsymbol{L}^{0}$ is the Levi subgroup of $\boldsymbol{P}^{0}$ containing $\boldsymbol{T}^{0}$. The duality thus defined coincides with the duality defined in [DM 1994, définition 3.10] when $\sigma$ is in $\left(\boldsymbol{G}^{1}\right)^{F}$.

In our context we can define $\mathrm{St}_{\boldsymbol{G}^{1}}$ similarly to [DM 1994, définition 3.16], as $D_{G^{1}}\left(\operatorname{Id}_{G^{1}}\right)$, and [DM 1994, proposition 3.18] remains true:

Proposition 2.13. $\mathrm{St}_{G^{1}}$ vanishes outside quasi-semisimple elements, and if $x \in$ $\left(\boldsymbol{G}^{1}\right)^{F}$ is quasi-semisimple, we have

$$
\mathrm{St}_{\boldsymbol{G}^{1}}(x)=\varepsilon_{\boldsymbol{G}^{1}} \varepsilon_{\left(\boldsymbol{G}^{x}\right)^{0}}\left|\left(\boldsymbol{G}^{x}\right)^{0}\right|_{p} .
$$

\section{A global formula for the scalar product of Deligne-Lusztig characters}

In this section we give a result of a different flavor, where we do not restrict our attention to a connected component $\boldsymbol{G}^{1}$.

Definition 3.1. For any character $\theta$ of $\boldsymbol{T}^{F}$, we define $R_{\boldsymbol{T}}^{\boldsymbol{G}}$ as in [DM 1994, définition 2.2]. If for a "torus" $\boldsymbol{T}$ and $\alpha=g \boldsymbol{G}^{0} \in \boldsymbol{G} / \boldsymbol{G}^{0}$ we denote by $\boldsymbol{T}^{[\alpha]}$ or $\boldsymbol{T}^{[g]}$ the unique connected component of $\boldsymbol{T}$ which meets $g \boldsymbol{G}^{0}$, this is equivalent to

$$
R_{\boldsymbol{T}}^{\boldsymbol{G}}(\theta)(g)=\left|\left(\boldsymbol{T}^{0}\right)^{F}\right| /\left|\boldsymbol{T}^{F}\right| \sum_{\left\{a \in \left[\boldsymbol{G}^{F} /\left.\left(\boldsymbol{G}^{0}\right)^{F}\right|^{a}\right.\right.}{ }_{\left.g \in \boldsymbol{T}^{F}\left(\boldsymbol{G}^{0}\right)^{F}\right\}} R_{\boldsymbol{T}^{\left[{ }^{a} g\right]}}^{\left.\boldsymbol{G}^{a} g\right]}(\theta)\left({ }^{a} g\right)
$$

for $g \in \boldsymbol{G}^{F}$, where the right-hand side is defined by Definition 2.4 (see [DM 1994, proposition 2.3]). 
We deduce from Proposition 2.8 the following formula for the whole group $\boldsymbol{G}$ :

Proposition 3.2. Let $\boldsymbol{T}, \boldsymbol{T}^{\prime}$ be two "tori" of $\boldsymbol{G}$ and let $\theta \in \operatorname{Irr}\left(\boldsymbol{T}^{F}\right), \theta^{\prime} \in \operatorname{Irr}\left(\boldsymbol{T}^{\prime F}\right)$. Then $\left\langle R_{\boldsymbol{T}}^{\boldsymbol{G}}(\theta), R_{\boldsymbol{T}^{\prime}}^{\boldsymbol{G}}\left(\theta^{\prime}\right)\right\rangle_{\boldsymbol{G}^{F}}=0$ if $\boldsymbol{T}^{0}$ and $\boldsymbol{T}^{\prime 0}$ are not $\boldsymbol{G}^{F}$-conjugate, and if $\boldsymbol{T}^{0}=\boldsymbol{T}^{\prime 0}$, we have

$$
\left\langle R_{\boldsymbol{T}}^{\boldsymbol{G}}(\theta), R_{\boldsymbol{T}^{\prime}}^{\boldsymbol{G}}\left(\theta^{\prime}\right)\right\rangle_{\boldsymbol{G}^{F}}=\left\langle\operatorname{Ind}_{\boldsymbol{T}^{F}}^{N_{G}\left(\boldsymbol{T}^{0}\right)^{F}}(\theta), \operatorname{Ind}_{\boldsymbol{T}^{\prime F}}^{N_{\boldsymbol{G}}\left(\boldsymbol{T}^{0}\right)^{F}}\left(\theta^{\prime}\right)\right\rangle_{N_{\boldsymbol{G}}\left(\boldsymbol{T}^{0}\right)^{F}} .
$$

Proof. Definition 3.1 can be written

$$
R_{\boldsymbol{T}}^{\boldsymbol{G}}(\theta)(g)=\left|\left(\boldsymbol{T}^{0}\right)^{F}\right| /\left|\boldsymbol{T}^{F}\right| \sum_{\left\{\left.a \in\left[\boldsymbol{G}^{F} /\left(\boldsymbol{G}^{0}\right)^{F}\right]\right|^{a}\right.} \boldsymbol{g}_{\left.g \in \boldsymbol{T}^{F}\left(\boldsymbol{G}^{0}\right)^{F}\right\}} R_{\left(a^{-1} \boldsymbol{T}\right)^{[g]}}^{\boldsymbol{G}^{[g]}}\left(a^{-1} \theta\right)(g) .
$$

So the scalar product we want to compute is equal to

$$
\begin{aligned}
&\left\langle R_{\boldsymbol{T}}^{\boldsymbol{G}}(\theta), R_{\boldsymbol{T}^{\prime}}^{\boldsymbol{G}}\left(\theta^{\prime}\right)\right\rangle_{\boldsymbol{G}^{F}}=\frac{1}{\left|\boldsymbol{G}^{F}\right|} \frac{\left|\left(\boldsymbol{T}^{0}\right)^{F}\right|}{\left|\boldsymbol{T}^{F}\right|} \frac{\left|\left(\boldsymbol{T}^{\prime 0}\right)^{F}\right|}{\left|\boldsymbol{T}^{\prime F}\right|} \\
& \times \sum_{\substack{\alpha \in \boldsymbol{G}^{F} / \boldsymbol{G}^{0} \\
g \in\left(\boldsymbol{G}^{0}\right)^{F} . \alpha}} \sum R_{\left(a^{-1} \boldsymbol{T}\right)^{[\alpha]}}^{\boldsymbol{G} . \alpha}\left(a^{-1} \theta\right)(g) \frac{R_{\left(a^{\prime-1} \boldsymbol{T}^{\prime}\right)^{[\alpha]}}^{\boldsymbol{G} . \alpha}\left(a^{\prime-1} \theta^{\prime}\right)(g)}{{ }^{\prime}},
\end{aligned}
$$

where the inner sum runs over $a \in\left[\boldsymbol{G}^{F} /\left(\boldsymbol{G}^{0}\right)^{F}\right]$ such that ${ }^{a} \alpha \in \boldsymbol{T}^{F}\left(\boldsymbol{G}^{0}\right)^{F}$ and $a^{\prime} \in\left[\boldsymbol{G}^{F} /\left(\boldsymbol{G}^{0}\right)^{F}\right]$ such that ${ }^{a^{\prime}} \alpha \in \boldsymbol{T}^{\prime F}\left(\boldsymbol{G}^{0}\right)^{F}$. This product can be written

$$
\begin{aligned}
\left\langle R_{\boldsymbol{T}}^{\boldsymbol{G}}(\theta), R_{\boldsymbol{T}^{\prime}}^{\boldsymbol{G}}\left(\theta^{\prime}\right)\right\rangle_{\boldsymbol{G}^{F}=}= & \frac{\left|\left(\boldsymbol{G}^{0}\right)^{F}\right|}{\left|\boldsymbol{G}^{F}\right|} \frac{\left|\left(\boldsymbol{T}^{0}\right)^{F}\right|}{\left|\boldsymbol{T}^{F}\right|} \frac{\left|\left(\boldsymbol{T}^{\prime 0}\right)^{F}\right|}{\left|\boldsymbol{T}^{\prime F}\right|} \\
& \times \sum_{\alpha \in \boldsymbol{G}^{F} / \boldsymbol{G}^{0}} \sum\left\langle R_{\left(a^{-1} \boldsymbol{T}\right)^{[\alpha]}}^{\boldsymbol{G} . \alpha}\left(a^{-1} \theta\right), R_{\left(a^{\prime-1} \boldsymbol{T}^{\prime}\right)^{[\alpha]}}^{\boldsymbol{G} . \alpha}\left(a^{\prime-1} \theta^{\prime}\right)\right\rangle_{\left(\boldsymbol{G}^{0}\right)^{F} . \alpha},
\end{aligned}
$$

where the inner sum is as above. By Proposition 2.8 the scalar product on the righthand side is zero unless $\left(a^{-1} \boldsymbol{T}\right)^{[\alpha]}$ and $\left({ }^{a^{\prime-1}} \boldsymbol{T}^{\prime}\right)^{[\alpha]}$ are $\left(\boldsymbol{G}^{0}\right)^{F}$-conjugate, which implies that $\boldsymbol{T}^{0}$ and $\boldsymbol{T}^{\prime 0}$ are $\left(\boldsymbol{G}^{0}\right)^{F}$-conjugate. So we can assume that $\boldsymbol{T}^{0}=\boldsymbol{T}^{\prime 0}$. Moreover for each $a^{\prime}$ indexing a nonzero summand, there is a representative $y \in a^{\prime-1}\left(\boldsymbol{G}^{0}\right)^{F}$ such that $\left({ }^{y} \boldsymbol{T}^{\prime}\right)^{[\alpha]}=\left({ }^{-1} \boldsymbol{T}\right)^{[\alpha]}$. This last equality and the condition on $a$ imply the condition ${ }^{a^{\prime}} \alpha \in \boldsymbol{T}^{\prime F}\left(\boldsymbol{G}^{0}\right)^{F}$ since this condition can be written $\left({ }^{y} \boldsymbol{T}^{\prime}\right)^{[\alpha]} \neq \varnothing$. Thus we can do the summation over all such $y \in \boldsymbol{G}^{F}$, provided we divide by $\left|N_{\left(\boldsymbol{G}^{0}\right)^{F}}\left(\left({ }^{a^{-1}} \boldsymbol{T}\right)^{[\alpha]}\right)\right|$. So we get, applying Proposition 2.8, that the above expression is equal to

$$
\begin{aligned}
& \frac{\left|\left(\boldsymbol{G}^{0}\right)^{F}\right|}{\left|\boldsymbol{G}^{F}\right|} \frac{\left|\left(\boldsymbol{T}^{0}\right)^{F}\right|^{2}}{\left|\boldsymbol{T}^{F}\right|\left|\boldsymbol{T}^{\prime F}\right|} \sum_{\alpha \in \boldsymbol{G}^{F} / \boldsymbol{G}^{0}} \sum_{\left\{\left.a \in\left[\boldsymbol{G}^{F} /\left(\boldsymbol{G}^{0}\right)^{F}\right]\right|^{a} \alpha \in \boldsymbol{T}^{F}\left(\boldsymbol{G}^{0}\right)^{F}\right\}}\left|N_{\left(\boldsymbol{G}^{0}\right)^{F}}\left(\left(a^{-1} \boldsymbol{T}\right)^{[\alpha]}\right)\right|^{-1} \\
& \times \sum_{\left\{y \in \boldsymbol{G}^{F} \mid\left(y \boldsymbol{T}^{\prime}\right)^{[\alpha]}=\left(a^{-1} \boldsymbol{T}\right)^{[\alpha]}\right\}}\left\langle\operatorname{Ind}_{\left(a^{-1} \boldsymbol{T}\right)^{[\alpha]}}^{N_{G^{0}}\left(a^{a^{-1}} \boldsymbol{T}^{0}\right)^{F}} a^{-1} \theta, \operatorname{Ind}_{\left(a^{-1} \boldsymbol{T}\right)^{[\alpha]}}^{N_{\boldsymbol{G}^{0} . .}\left(a^{-1} \boldsymbol{T}^{0}\right)^{F}} y^{\prime}\right\rangle_{N_{\boldsymbol{G}^{0 . \alpha}}\left(\boldsymbol{T}^{0}\right)^{F}} .
\end{aligned}
$$


We now conjugate everything by $a$, take $a y$ as new variable $y$, set $b={ }^{a} \alpha$ and get

$$
\begin{aligned}
\frac{\left|\left(\boldsymbol{T}^{0}\right)^{F}\right|^{2}}{\left|\boldsymbol{T}^{F}\right| \mid \boldsymbol{T}^{\prime F \mid}} & \sum_{b \in \boldsymbol{T}^{F} /\left(\boldsymbol{T}^{0}\right)^{F}}\left|N_{\left(\boldsymbol{G}^{0}\right)^{F}}\left(\boldsymbol{T}^{[b]}\right)\right|^{-1} \\
& \times \sum_{\left\{y \in \boldsymbol{G}^{F} \mid\left({ }^{y} \boldsymbol{T}^{\prime}\right)^{[b]}=\boldsymbol{T}^{[b]\}}\right.}\left\langle\operatorname{Ind}_{\boldsymbol{T}^{[b] F}}^{N_{\boldsymbol{G}^{0}, b}\left(\boldsymbol{T}^{0}\right)^{F}} \theta, \operatorname{Ind}_{\boldsymbol{T}^{[b] F}}^{N_{\boldsymbol{G}^{0}, b}\left(\boldsymbol{T}^{0}\right)^{F}} y^{\prime}\right\rangle_{N_{\boldsymbol{G}^{0 . b}}\left(\boldsymbol{T}^{0}\right)^{F}}
\end{aligned}
$$

since for $b \in \boldsymbol{T}^{F} /\left(\boldsymbol{T}^{0}\right)^{F}$, any choice of $a \in \boldsymbol{G}^{F} /\left(\boldsymbol{G}^{0}\right)^{F}$ gives an $\alpha={ }^{a^{-1}} b$ which satisfies the condition ${ }^{a} \alpha \in \boldsymbol{T}^{F}\left(\boldsymbol{G}^{0}\right)^{F}$.

Let us now transform the right-hand side of Proposition 3.2. Using the definition we have

$$
\begin{aligned}
& \left\langle\operatorname{Ind}_{\boldsymbol{T}^{F}}^{N_{\boldsymbol{G}}\left(\boldsymbol{T}^{0}\right)^{F}}(\theta), \operatorname{Ind}_{\boldsymbol{T}^{\prime F}}^{N_{\boldsymbol{G}}\left(\boldsymbol{T}^{0}\right)^{F}}(\theta)\right\rangle_{N_{\boldsymbol{G}}\left(\boldsymbol{T}^{0}\right)^{F}} \\
& =\left|\boldsymbol{T}^{F}\right|^{-1}\left|\boldsymbol{T}^{\prime F}\right|^{-1}\left|N_{\boldsymbol{G}}\left(\boldsymbol{T}^{0}\right)^{F}\right|^{-1} \sum_{\left\{n, x, x^{\prime} \in N_{\boldsymbol{G}}\left(\boldsymbol{T}^{0}\right)^{F} \mid{ }^{x} n \in \boldsymbol{T},{ }^{x^{\prime}} n \in \boldsymbol{T}^{\prime}\right\}} \theta\left({ }^{x} n\right) \overline{\theta^{\prime}\left({ }^{x^{\prime}} n\right)} \\
& =\left|\boldsymbol{T}^{F}\right|^{-1}\left|\boldsymbol{T}^{\prime F}\right|^{-1}\left|N_{\boldsymbol{G}}\left(\boldsymbol{T}^{0}\right)^{F}\right|^{-1}
\end{aligned}
$$

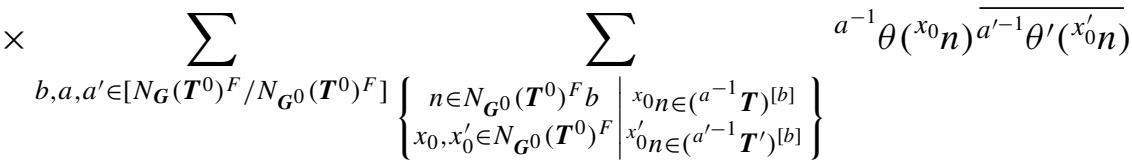

$$
\begin{aligned}
& =\frac{\left|\left(\boldsymbol{T}^{0}\right)^{F}\right|}{\left|\boldsymbol{T}^{F}\right|} \frac{\left|\left(\boldsymbol{T}^{\prime 0}\right)^{F}\right|}{\left|\boldsymbol{T}^{\prime F}\right|} \frac{\left|N_{\boldsymbol{G}^{0}}\left(\boldsymbol{T}^{0}\right)^{F}\right|}{\left|N_{\boldsymbol{G}}\left(\boldsymbol{T}^{0}\right)^{F}\right|} \\
& \times \sum_{b, a, a^{\prime} \in\left[N_{\boldsymbol{G}}\left(\boldsymbol{T}^{0}\right)^{F} / N_{\boldsymbol{G}^{0}}\left(\boldsymbol{T}^{0}\right)^{F}\right]}\left\langle\operatorname{Ind}_{\left(a^{-1} \boldsymbol{T}\right)^{[b] F}}^{N_{G^{0}}\left(\boldsymbol{T}^{0}\right)^{F} \cdot b} a^{-1} \theta, \operatorname{Ind}_{\left(a^{\prime-1} \boldsymbol{T}^{\prime}\right)^{[b] F}}^{N_{\boldsymbol{G}^{0}}\left(\boldsymbol{T}^{0}\right)^{F} \cdot b} a^{\prime-1} \theta^{\prime}\right\rangle_{N_{\boldsymbol{G}^{0}}\left(\boldsymbol{T}^{0}\right)^{F} b} .
\end{aligned}
$$

We may simplify the sum by conjugating the terms in the scalar product by $a$ to get

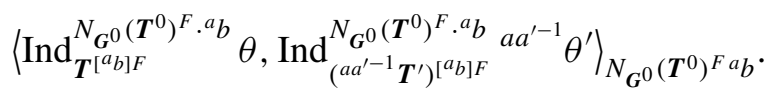

Then we may take, given $a$, the conjugate ${ }^{a} b$ as new variable $b$, and $a a^{\prime-1}$ as the new variable $a^{\prime}$ to get

$$
\frac{\left|\left(\boldsymbol{T}^{0}\right)^{F}\right|}{\left|\boldsymbol{T}^{F}\right|} \frac{\left|\left(\boldsymbol{T}^{\prime 0}\right)^{F}\right|}{\left|\boldsymbol{T}^{\prime F}\right|} \sum_{b, a^{\prime} \in\left[\frac{N_{\boldsymbol{G}}\left(\boldsymbol{T}^{0}\right)^{F}}{\left.N_{\boldsymbol{G}^{0}} \boldsymbol{T}^{0}\right)^{F}}\right]}\left\langle\operatorname{Ind}_{\boldsymbol{T}^{[b] F}}^{N_{\boldsymbol{G}^{0}}\left(\boldsymbol{T}^{0}\right)^{F} \cdot b} \theta, \operatorname{Ind}_{\left(a^{\prime} \boldsymbol{T}^{\prime}\right)^{[b] F}}^{N_{\boldsymbol{B}^{0}}\left(\boldsymbol{T}^{0}\right)^{F} \cdot b} a^{\prime} \theta^{\prime}\right\rangle_{N_{\boldsymbol{G}^{0}}\left(\boldsymbol{T}^{0}\right)^{F} b} .
$$

Now, by Frobenius reciprocity, for the inner scalar product not to vanish, there must be some element $x \in N_{\boldsymbol{G}^{0}}\left(\boldsymbol{T}^{0}\right)^{F}$ such that ${ }^{x}\left({ }^{a^{\prime}} \boldsymbol{T}^{\prime}\right)^{[b] F}$ meets $\boldsymbol{T}^{[b] F}$ which, considering the definitions, implies that $\left({ }^{x a^{\prime}} \boldsymbol{T}^{\prime}\right)^{[b]}=\boldsymbol{T}^{[b]}$. We may then conjugate 
the term

$$
\operatorname{Ind}_{\left(a^{\prime} \boldsymbol{T}^{\prime}\right)^{[b] F}}^{N_{\boldsymbol{G}^{0}}\left(\boldsymbol{T}^{0}\right)^{F} \cdot b} a^{\prime} \theta^{\prime}
$$

by such an $x$ to get

$$
\operatorname{Ind}_{\boldsymbol{T}^{b] F}}^{N_{\boldsymbol{G}^{0}}\left(\boldsymbol{T}^{0}\right)^{F} \cdot b} x a^{\prime} \theta^{\prime}
$$

and take $y=x a^{\prime}$ as a new variable, provided we count the number of $x$ for a given $a^{\prime}$, which is $\left|N_{\boldsymbol{G}^{0}}\left(\boldsymbol{T}^{[b]}\right)^{F}\right|$. We get

$$
\begin{aligned}
& \frac{\left|\left(\boldsymbol{T}^{0}\right)^{F}\right|}{\left|\boldsymbol{T}^{F}\right|} \frac{\left|\left(\boldsymbol{T}^{\prime 0}\right)^{F}\right|}{\left|\boldsymbol{T}^{\prime F}\right|} \sum_{b \in\left[N_{\boldsymbol{G}}\left(\boldsymbol{T}^{0}\right)^{F} / N_{\boldsymbol{G}^{0}}\left(\boldsymbol{T}^{0}\right)^{F}\right]}\left|N_{\boldsymbol{G}^{0}}\left(\boldsymbol{T}^{[b]}\right)^{F}\right|^{-1} \\
& \quad \times \sum_{\left\{y \in N_{\boldsymbol{G}}\left(\boldsymbol{T}^{0}\right)^{F} \mid\left({ }^{y} \boldsymbol{T}^{\prime}\right)^{[b]}=\boldsymbol{T}^{[b]}\right\}}\left\langle\operatorname{Ind}_{\boldsymbol{T}^{b] F}}^{N_{\boldsymbol{G}^{0}}\left(\boldsymbol{T}^{0}\right)^{F} \cdot b} \theta, \operatorname{Ind}_{\boldsymbol{T}^{[b] F}}^{N_{\boldsymbol{G}^{0}}\left(\boldsymbol{T}^{0}\right)^{F} \cdot b} y^{y} \theta_{N_{\boldsymbol{G}^{0}}\left(\boldsymbol{T}^{0}\right)^{F} b^{*}}\right.
\end{aligned}
$$

Since any $b \in\left[N_{\boldsymbol{G}}\left(\boldsymbol{T}^{0}\right)^{F} / N_{\boldsymbol{G}^{0}}\left(\boldsymbol{T}^{0}\right)^{F}\right]$ such that $\boldsymbol{T}^{[b] F}$ is not empty has a representative in $\boldsymbol{T}^{F}$, we can do the first summation over $b \in\left[\boldsymbol{T}^{F} /\left(\boldsymbol{T}^{0}\right)^{F}\right]$ so that (3.3) is equal to (3.4).

\section{Counting unipotent elements in disconnected groups}

A proof of the following result appeared recently in [Lawther et al. 2014, Theorem 1.1]; our proof given below, that we wrote in February 1994 in answer to a question of Cheryl Praeger, is much shorter and case-free.

Proposition 4.1. Assume $\boldsymbol{G}^{1} / \boldsymbol{G}^{0}$ is unipotent and take $\sigma \in \boldsymbol{G}^{1}$ unipotent $F$-stable and quasicentral (see Lemma 2.2). Then the number of unipotent elements of $\left(G^{1}\right)^{F}$ is given by $\left|\left(\boldsymbol{G}^{\sigma 0}\right)^{F}\right|_{p}^{2}\left|\boldsymbol{G}^{0^{F}}\right| /\left|\left(\boldsymbol{G}^{\sigma 0}\right)^{F}\right|$.

Proof. Let $\chi_{\mathcal{U}}$ be the characteristic function of the set of unipotent elements of $\left(G^{1}\right)^{F}$. Then $\left|\left(\boldsymbol{G}^{1}\right)_{\text {unip }}^{F}\right|=\left|\left(\boldsymbol{G}^{1}\right)^{F}\right|\left\langle\chi_{\mathcal{U}}, \mathrm{Id}\right\rangle_{\left(\boldsymbol{G}^{1}\right)^{F}}$ and

$$
\left\langle\chi_{\mathcal{U}}, \mathrm{Id}\right\rangle_{\left(G^{1}\right)^{F}}=\left\langle\mathrm{D}_{\boldsymbol{G}^{1}}\left(\chi_{\mathcal{U}}\right), \mathrm{D}_{\boldsymbol{G}^{1}}(\mathrm{Id})\right\rangle_{\left(\boldsymbol{G}^{1}\right)^{F}}=\left\langle\mathrm{D}_{\boldsymbol{G}^{1}}\left(\chi_{\mathcal{U}}\right), \mathrm{St}_{\boldsymbol{G}^{1}}\right\rangle_{\left(\boldsymbol{G}^{1}\right)^{F}},
$$

where the first equality holds since $\mathrm{D}_{\boldsymbol{G}^{1}}$ is an isometry by [DM 1994, corollaire 3.12]. According to [DM 1994, proposition 2.11], for any $\sigma$-stable and $F$-stable Levi subgroup $\boldsymbol{L}^{0}$ of a $\sigma$-stable parabolic subgroup of $\boldsymbol{G}^{0}$, setting $\boldsymbol{L}^{1}=\boldsymbol{L}^{0} . \sigma$, we have $R_{\boldsymbol{L}^{1}}^{\boldsymbol{G}^{1}}\left(\left.\pi \cdot \chi_{\mathcal{U}}\right|_{\left(\boldsymbol{L}^{1}\right)^{F}}\right)=R_{\boldsymbol{L}^{1}}^{\boldsymbol{G}^{1}}(\pi) \cdot \chi_{\mathcal{U}}$ and $\left.{ }^{*} R_{\boldsymbol{L}^{1}}^{\boldsymbol{G}^{1}}(\varphi) \cdot \chi_{\mathcal{U}}\right|_{\left(\boldsymbol{L}^{1}\right)^{F}}={ }^{*} R_{\boldsymbol{L}^{1}}^{\boldsymbol{G}^{1}}\left(\varphi \cdot \chi_{\mathcal{U}}\right)$. Thus, by (2.12), $\mathrm{D}_{G^{1}}\left(\pi \cdot \chi_{\mathcal{U}}\right)=\mathrm{D}_{G^{1}}(\pi) \cdot \chi_{\mathcal{U}}$; in particular, $\mathrm{D}_{G^{1}}\left(\chi_{\mathcal{U}}\right)=\mathrm{D}_{G^{1}}(\mathrm{Id}) \cdot \chi_{\mathcal{U}}=\mathrm{St}_{G^{1}} \cdot \chi_{\mathcal{U}}$. Now, by Proposition 2.13, the only unipotent elements on which $\mathrm{St}_{\boldsymbol{G}^{1}}$ does not vanish are the quasi-semisimple (thus quasicentral) ones; by [DM 1994, corollaire 1.37], all such elements are in the $\boldsymbol{G}^{0^{F}}$-class of $\sigma$ and, again by Proposition 2.13, we have 
$\mathrm{St}_{\boldsymbol{G}^{1}}(\sigma)=\left|\left(\boldsymbol{G}^{\sigma 0}\right)^{F}\right|_{p}$. We get

$$
\begin{aligned}
\left|\left(\boldsymbol{G}^{1}\right)^{F}\right|\left\langle\mathrm{D}_{\boldsymbol{G}^{1}}\left(\chi_{\mathcal{U}}\right), \mathrm{St}_{\boldsymbol{G}^{1}}\right\rangle_{\left(\boldsymbol{G}^{1}\right)^{F}} & =\left|\left(\boldsymbol{G}^{1}\right)^{F}\right|\left\langle\mathrm{St}_{\boldsymbol{G}^{1}} \cdot \chi_{\mathcal{U}}, \mathrm{St}_{\boldsymbol{G}^{1}}\right\rangle_{\left(\boldsymbol{G}^{1}\right)^{F}} \\
& =\mid\left.\left\{\boldsymbol{G}^{0 F} \text {-class of } \sigma\right\}||\left(\boldsymbol{G}^{\sigma 0}\right)^{F}\right|_{p} ^{2},
\end{aligned}
$$

whence the proposition.

Example 4.2. The formula of Proposition 4.1 applies in the following cases where $\sigma$ induces a diagram automorphism of order 2 and $q$ is a power of 2 :

- $\boldsymbol{G}^{0}=\mathrm{SO}_{2 n},\left(\boldsymbol{G}^{\sigma 0}\right)^{F}=\mathrm{SO}_{2 n-1}\left(\mathbb{F}_{q}\right)$;

- $\boldsymbol{G}^{0}=\mathrm{GL}_{2 n},\left(\boldsymbol{G}^{\sigma 0}\right)^{F}=\operatorname{Sp}_{2 n}\left(\mathbb{F}_{q}\right)$;

- $\boldsymbol{G}^{0}=\mathrm{GL}_{2 n+1},\left(\boldsymbol{G}^{\sigma 0}\right)^{F}=\mathrm{SO}_{2 n+1}\left(\mathbb{F}_{q}\right) \simeq \operatorname{Sp}_{2 n}\left(\mathbb{F}_{q}\right)$;

- $\boldsymbol{G}^{0}=E_{6},\left(\boldsymbol{G}^{\sigma 0}\right)^{F}=F_{4}\left(\mathbb{F}_{q}\right)$;

And it applies to the case where $\boldsymbol{G}^{0}=\operatorname{Spin}_{8}$, where $\sigma$ induces a diagram automorphism of order 3 and $q$ is a power of 3 , in which case $\left(\boldsymbol{G}^{\sigma 0}\right)^{F}=G_{2}\left(\mathbb{F}_{q}\right)$.

\section{Tensoring by the Steinberg character}

Proposition 5.1. Let $\boldsymbol{L}^{1}$ be an $F$-stable Levi of $\boldsymbol{G}^{1}$. Then, for any class function $\gamma$ on $\left(\boldsymbol{G}^{1}\right)^{F}$, we have

$$
{ }^{*} R_{\boldsymbol{L}^{1}}^{\boldsymbol{G}^{1}}\left(\gamma \cdot \varepsilon_{\boldsymbol{G}^{1}} \mathrm{St}_{\boldsymbol{G}^{1}}\right)=\varepsilon_{\boldsymbol{L}^{1}} \operatorname{St}_{\boldsymbol{L}^{1}} \operatorname{Res}_{\left(\boldsymbol{L}^{1}\right)^{F}}^{\left(\boldsymbol{G}^{1}\right)^{F}} \gamma
$$

Proof. Let $s u$ be the Jordan decomposition of a quasi-semisimple element of $\boldsymbol{G}^{1}$ with $s$ semisimple. We claim that $u$ is quasicentral in $\boldsymbol{G}^{s}$. Indeed $s u$, being quasi-semisimple, is in a "torus" $\boldsymbol{T}$; thus $s$ and $u$ also are in $\boldsymbol{T}$. By [DM 1994, théorème 1.8(iii)], the intersection of $\boldsymbol{T} \cap \boldsymbol{G}^{s}$ is a "torus" of $\boldsymbol{G}^{s}$; thus $u$ is quasisemisimple in $\boldsymbol{G}^{s}$, and hence quasicentral since unipotent.

Let $t v$ be the Jordan decomposition of an element $l \in\left(\boldsymbol{L}^{1}\right)^{F}$, where $t$ is semisimple. Since $\mathrm{St}_{\boldsymbol{L}^{1}}$ vanishes outside quasi-semisimple elements, the right-hand side of the proposition vanishes on $l$ unless it is quasi-semisimple, which by our claim means that $v$ is quasicentral in $\boldsymbol{L}^{t}$. By the character formula Proposition 2.5 the left-hand side of the proposition evaluates at $l$ to

$$
{ }^{*} R_{\boldsymbol{L}^{1}}^{\boldsymbol{G}^{1}}\left(\gamma \cdot \varepsilon_{\boldsymbol{G}^{1}} \mathrm{St}_{\boldsymbol{G}^{1}}\right)(l)=\left|\left(\boldsymbol{G}^{t 0}\right)^{F}\right|^{-1} \sum_{u \in\left(\boldsymbol{G}^{t 0} \cdot v\right)_{\text {unip }}^{F}} Q_{\boldsymbol{L}^{t 0}}^{\boldsymbol{G}^{t 0}}\left(u, v^{-1}\right) \gamma(t u) \varepsilon_{\boldsymbol{G}^{1}} \mathrm{St}_{\boldsymbol{G}^{1}}(t u) .
$$

By the same argument as above, applied to $\mathrm{St}_{G^{1}}$, the only nonzero terms in the above sum are for $u$ quasicentral in $\boldsymbol{G}^{t}$. For such $u$, by [DM 1994, proposition 4.16], $Q_{\boldsymbol{L}^{t 0}}^{\boldsymbol{G}^{t 0}}\left(u, v^{-1}\right)$ vanishes unless $u$ and $v$ are $\left(\boldsymbol{G}^{t 0}\right)^{F}$-conjugate. Hence both sides of the equality-to-prove vanish unless $u$ and $v$ are quasicentral and $\left(\boldsymbol{G}^{t 0}\right)^{F}$-conjugate. In that case, by [DM 1994, proposition 4.16] and [Digne and Michel 1991, (**), 


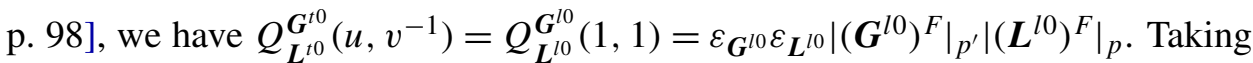
into account that the $\left(\boldsymbol{G}^{t 0}\right)^{F}$-class of $v$ has cardinality $\left|\left(\boldsymbol{G}^{t 0}\right)^{F}\right| /\left|\left(\boldsymbol{G}^{l 0}\right)^{F}\right|$ and that by Proposition 2.13 we have $\operatorname{St}_{\boldsymbol{G}^{1}}(l)=\varepsilon_{\boldsymbol{G}^{\sigma 0}} \varepsilon_{\boldsymbol{G}^{l 0}}\left|\left(\boldsymbol{G}^{l 0}\right)^{F}\right|_{p}$, the left-hand side of the proposition reduces to $\gamma(l) \varepsilon_{\boldsymbol{L}^{l 0}}\left|\left(\boldsymbol{L}^{l 0}\right)^{F}\right|_{p}$, which is also the value of the right-hand side by applying Proposition 2.13 in $\boldsymbol{L}^{1}$.

By adjunction, we get the following:

Corollary 5.2. For any class function $\lambda$ on $\left(\boldsymbol{L}^{1}\right)^{F}$, we have

$$
R_{\boldsymbol{L}^{1}}^{\boldsymbol{G}^{1}}(\lambda) \varepsilon_{\boldsymbol{G}^{1}} \operatorname{St}_{\boldsymbol{G}^{1}}=\operatorname{Ind}_{\left(\boldsymbol{L}^{1}\right)^{F}}^{\left(\boldsymbol{G}^{1}\right)^{F}}\left(\varepsilon_{\boldsymbol{L}^{1}} \mathrm{St}_{\boldsymbol{L}^{1}} \lambda\right) .
$$

\section{Characteristic functions of quasi-semisimple classes}

One of the goals of this section is Proposition 6.4 where we give a formula for the characteristic function of a quasi-semisimple class which shows, in particular, that it is uniform; this generalizes the case of quasicentral elements given in [DM 1994, proposition 4.14].

If $x \in\left(\boldsymbol{G}^{1}\right)^{F}$ has Jordan decomposition $x=s u$, we will denote by $d_{x}$ the map from class functions on $\left(\boldsymbol{G}^{1}\right)^{F}$ to class functions on $\left(C_{\boldsymbol{G}}(s)^{0} \cdot u\right)^{F}$ given by

$$
\left(d_{x} f\right)(v)= \begin{cases}f(s v) & \text { if } v \in\left(C_{\boldsymbol{G}}(s)^{0} \cdot u\right)^{F} \text { is unipotent } \\ 0 & \text { otherwise. }\end{cases}
$$

Lemma 6.1. Let $\boldsymbol{L}^{1}$ be an $F$-stable Levi of $\boldsymbol{G}^{1}$. If $x=$ su is the Jordan decomposition of an element of $\left(\boldsymbol{L}^{1}\right)^{F}$, we have $d_{x} \circ{ }^{*} R_{\boldsymbol{L}^{1}}^{G^{1}}={ }^{*} R_{C_{\boldsymbol{L}}(s)^{0} \cdot u}^{C_{G}(s)} \circ d_{x}$.

Proof. For $v$ unipotent in $\left(C_{\boldsymbol{G}}(s)^{0} \cdot u\right)^{F}$ and $f$ a class function on $\left(\boldsymbol{G}^{1}\right)^{F}$, we have

$$
\left(d_{x}{ }^{*} R_{\boldsymbol{L}^{1}}^{G^{1}} f\right)(v)=\left({ }^{*} R_{\boldsymbol{L}^{1}}^{G^{1}} f\right)(s v)=\left({ }^{*} R_{C_{\boldsymbol{L}}(s)^{0} \cdot s u}^{C_{G}(s)} f\right)(s v)=\left({ }^{*} R_{C_{\boldsymbol{L}}(s)^{0} \cdot u}^{C_{G}(s)} d_{x} f\right)(v),
$$

where the second equality is by [DM 1994, corollaire 2.9] and the last is by the character formula Proposition 2.5(iii).

Proposition 6.2. If $x=s u$ is the Jordan decomposition of an element of $\left(\boldsymbol{G}^{1}\right)^{F}$, we have $d_{x} \circ p^{G^{1}}=p^{C_{G}(s)^{0} \cdot u} \circ d_{x}$.

Proof. Let $f$ be a class function on $\left(G^{1}\right)^{F}$. For $v \in\left(C_{G}(s)^{0} \cdot u\right)^{F}$ unipotent, we have

$$
\left(d_{x} p^{\boldsymbol{G}^{1}} f\right)(v)=p^{\boldsymbol{G}^{1}} f(s v)=\left|\left(\boldsymbol{G}^{1}\right)^{F}\right|^{-1} \sum_{\boldsymbol{T}^{1} \in \mathcal{T}_{1}^{F}}\left|\left(\boldsymbol{T}^{1}\right)^{F}\right|\left(R_{\boldsymbol{T}^{1}}^{\boldsymbol{G}^{1}}{ }^{*} R_{\boldsymbol{T}^{1}}^{\boldsymbol{G}^{1}} f\right)(s v),
$$

where the last equality is by Corollary 2.10 , and which by Proposition 2.5(ii) is

$$
\sum_{\boldsymbol{T}^{1} \in \mathcal{T}_{1}^{F}} \sum_{\left\{\left.h \in\left(\boldsymbol{G}^{0}\right)^{F}\right|^{h} \boldsymbol{T} \ni s\right\}} \frac{\left|{ }^{h} \boldsymbol{T}^{0} \cap C_{\boldsymbol{G}}(s)^{0 F}\right|}{\left|\left(\boldsymbol{G}^{0}\right)^{F}\right|\left|C_{\boldsymbol{G}}(s)^{0 F}\right|}\left(R_{h \boldsymbol{T} \cap C_{\boldsymbol{G}}(s)^{0} \cdot s u}^{C_{\boldsymbol{G}}(s)^{0} \cdot s u} \circ^{h *} R_{\boldsymbol{T}^{1}}^{\boldsymbol{G}^{1}} f\right)(s v) .
$$


Using that ${ }^{h *} R_{\boldsymbol{T}^{1}}^{\boldsymbol{G}^{1}} f={ }^{*} R_{h^{1}}^{G^{1}} f$ and summing over the ${ }^{h} \boldsymbol{T}^{1}$, this becomes

$$
\sum_{\left\{\boldsymbol{T}^{1} \in \mathcal{T}_{1}^{F} \mid \boldsymbol{T} \ni s\right\}} \frac{\left|\boldsymbol{T}^{0} \cap C_{\boldsymbol{G}}(s)^{0 F}\right|}{\left|C_{\boldsymbol{G}}(s)^{0 F}\right|}\left(R_{\boldsymbol{T}^{1} \cap C_{\boldsymbol{G}}(s)^{0} \cdot s u}^{C_{\boldsymbol{G}}(s)^{0} \cdot s u} \circ^{*} R_{\boldsymbol{T}^{1}}^{\boldsymbol{G}^{1}} f\right)(s v) .
$$

Using that by Proposition 2.5(i) for any class function $\chi$ on $T^{1} \cap C_{G}(s)^{0} \cdot s u^{F}$,

$$
\begin{aligned}
\left(R_{\boldsymbol{T}^{1} \cap C_{\boldsymbol{G}}(s)^{0} \cdot s u}^{C_{\boldsymbol{B}}(s)} \chi\right)(s v) & =\left|\boldsymbol{T}^{0} \cap C_{\boldsymbol{G}}(s)^{0 F}\right|^{-1} \sum_{v^{\prime} \in\left(\boldsymbol{T} \cap C_{\boldsymbol{G}}(s)^{0} \cdot u\right)_{\text {unip }}^{F}} Q_{\left(\boldsymbol{T}^{s}\right)^{0}}^{\left(\boldsymbol{G}^{s}\right)^{0}}\left(v, v^{-1}\right) \chi\left(s v^{\prime}\right) \\
& =R_{\boldsymbol{T} \cap C_{\boldsymbol{G}}(s)^{0} \cdot u}^{C_{\boldsymbol{G}}(s)^{0} \cdot u}\left(d_{x} \chi\right)(v),
\end{aligned}
$$

and using Lemma 6.1, we get

$$
\left|C_{\boldsymbol{G}}(s)^{0} \cdot s u^{F}\right|^{-1} \sum_{\left\{\boldsymbol{T}^{1} \in \mathcal{T}_{1}^{F} \mid \boldsymbol{T} \ni s\right\}}\left|\left(\boldsymbol{T}^{s}\right)^{0^{F}}\right|\left(R_{\boldsymbol{T} \cap C_{\boldsymbol{G}}(s)^{0} \cdot u}^{C_{\boldsymbol{G}}(s)}{ }^{0} \cdot{ }^{*} R_{\boldsymbol{T} \cap C_{\boldsymbol{G}}(s)^{0} \cdot u}^{C_{\boldsymbol{G}}(s)^{0} \cdot u} d_{x} f\right)(v),
$$

which is the desired result if we apply Corollary 2.10 in $C_{G}(s)^{0} \cdot u$ and remark that by [DM 1994, théorème 1.8(iv)], the map $\boldsymbol{T}^{1} \mapsto \boldsymbol{T} \cap C_{\boldsymbol{G}}(s)^{0} \cdot u$ induces a bijection between $\left\{\boldsymbol{T}^{1} \in \mathcal{T}_{1}^{F} \mid \boldsymbol{T} \ni s\right\}$ and $F$-stable "tori" of $C_{\boldsymbol{G}}(s)^{0} \cdot u$.

Corollary 6.3. A class function $f$ on $\left(G^{1}\right)^{F}$ is uniform if and only if for every $x \in\left(\boldsymbol{G}^{1}\right)^{F}$, the function $d_{x} f$ is uniform.

Proof. Indeed, $f=p^{\boldsymbol{G}^{1}} f$ if and only if for any $x \in\left(\boldsymbol{G}^{1}\right)^{F}$, we have $d_{x} f=$ $d_{x} p^{\boldsymbol{G}^{1}} f=p^{C_{\boldsymbol{G}}(s)^{0} \cdot u} d_{x} f$, where the last equality holds by Proposition 6.2.

For $x \in\left(G^{1}\right)^{F}$, we consider the class function $\pi_{x}^{G^{1}}$ on $\left(\boldsymbol{G}^{1}\right)^{F}$ defined by

$$
\pi_{x}^{\boldsymbol{G}^{1}}(y)= \begin{cases}0 & \text { if } y \text { is not conjugate to } x, \\ \left|C_{\boldsymbol{G}^{0}}(x)^{F}\right| & \text { if } y=x .\end{cases}
$$

Proposition 6.4. For a quasi-semisimple $x \in\left(\boldsymbol{G}^{1}\right)^{F}$, the function $\pi_{x}^{\boldsymbol{G}^{1}}$ is uniform, given by

$$
\begin{aligned}
\pi_{x}^{\boldsymbol{G}^{1}} & =\varepsilon_{\boldsymbol{G}^{x 0}}\left|C_{\boldsymbol{G}}(x)^{0}\right|_{p}^{-1} \sum_{\left\{\boldsymbol{T}^{1} \in \mathcal{T}_{1}^{F} \mid \boldsymbol{T}^{1} \ni x\right\}} \varepsilon_{\boldsymbol{T}^{1}} R_{\boldsymbol{T}^{1}}^{\boldsymbol{G}^{1}}\left(\pi_{x}^{\boldsymbol{T}^{1}}\right) \\
& =\left|W^{0}(x)\right|^{-1} \sum_{w \in W^{0}(x)} \operatorname{dim} R_{\boldsymbol{T}_{w}}^{C_{\boldsymbol{G}}(x)^{0}}(\mathrm{Id}) R_{C_{\boldsymbol{G}^{1}}\left(\boldsymbol{T}_{w}\right)}^{\boldsymbol{G}^{1}}\left(\pi_{x}^{C_{\boldsymbol{G}^{1}}\left(\boldsymbol{T}_{w}\right)}\right)
\end{aligned}
$$

where in the second equality $W^{0}(x)$ denotes the Weyl group of $C_{\boldsymbol{G}}(x)^{0}$ and $\boldsymbol{T}_{w}$ denotes an F-stable torus of type $w$ of this last group.

Proof. First, using Corollary 6.3 we prove that $\pi_{x}^{G^{1}}$ is uniform. Let $s u$ be the Jordan decomposition of $x$. For $y \in\left(\boldsymbol{G}^{1}\right)^{F}$, the function $d_{y} \pi_{x}^{\boldsymbol{G}^{1}}$ is zero unless the semisimple part of $y$ is conjugate to $s$. Hence it is sufficient to evaluate $d_{y} \pi_{x}^{G^{1}}(v)$ for elements $y$ whose semisimple part is equal to $s$. For such elements, $d_{y} \pi_{x}^{\boldsymbol{G}^{1}}(v)$ 
is up to a coefficient equal to $\pi_{u}^{C_{G}(s)^{0} \cdot u}$. This function is uniform by [DM 1994, proposition 4.14], since $u$ being the unipotent part of a quasi-semisimple element is quasicentral in $C_{G}(s)$ (see the beginning of the proof of Proposition 5.1).

Thus we have $\pi_{x}^{G^{1}}=p^{G^{1}} \pi_{x}^{G^{1}}$. We use this to get the formula of the proposition. We start by using Proposition 2.13 to write $\pi_{x}^{\boldsymbol{G}^{1}} \mathrm{St}_{\boldsymbol{G}^{1}}=\varepsilon_{\boldsymbol{G}^{1}} \varepsilon_{\boldsymbol{G}^{x 0}}\left|\left(\boldsymbol{G}^{x 0}\right)^{F}\right|_{p} \pi_{x}^{\boldsymbol{G}^{1}}$, or equivalently, $\pi_{x}^{\boldsymbol{G}^{1}}=\varepsilon_{\boldsymbol{G}^{1}} \varepsilon_{\boldsymbol{G}^{x 0}}\left|\left(\boldsymbol{G}^{x 0}\right)^{F}\right|_{p}^{-1} p^{\boldsymbol{G}^{1}}\left(\pi_{x}^{\boldsymbol{G}^{1}} \mathrm{St}_{\boldsymbol{G}^{1}}\right)$. Using Corollary 2.10

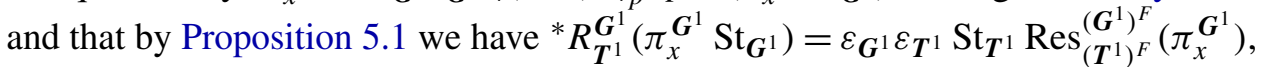
we get

$$
p^{\boldsymbol{G}^{1}}\left(\pi_{x}^{\boldsymbol{G}^{1}} \mathrm{St}_{\boldsymbol{G}^{1}}\right)=\varepsilon_{\boldsymbol{G}^{1}}\left|\left(\boldsymbol{G}^{1}\right)^{F}\right|^{-1} \sum_{\boldsymbol{T}^{1} \in \mathcal{T}_{1}^{F}}\left|\left(\boldsymbol{T}^{1}\right)^{F}\right| \varepsilon_{\boldsymbol{T}^{1}} R_{\boldsymbol{T}^{1}}^{\boldsymbol{G}^{1}}\left(\operatorname{St}_{\boldsymbol{T}^{1}} \operatorname{Res}_{\left(\boldsymbol{T}^{1}\right)^{F}}^{\left(\boldsymbol{G}^{1}\right)^{F}}\left(\pi_{x}^{\boldsymbol{G}^{1}}\right)\right) .
$$

The function $\mathrm{St}_{T^{1}}$ is constant equal to 1 . Now we have

$$
\operatorname{Res}_{\left(\boldsymbol{T}^{1}\right)^{F}}^{\left(\boldsymbol{G}^{1}\right)^{F}} \pi_{x}^{\boldsymbol{G}^{1}}=\left|\left(\boldsymbol{T}^{0}\right)^{F}\right|^{-1} \sum_{\left\{g \in\left(\boldsymbol{G}^{0}\right)^{F} \mid g_{x} \in \boldsymbol{T}^{1}\right\}} \pi_{g_{x}}^{\boldsymbol{T}^{1}} .
$$

To see this, do the scalar product with a class function $f$ on $\left(\boldsymbol{T}^{1}\right)^{F}$ :

$$
\left\langle\operatorname{Res}_{\left(\boldsymbol{T}^{1}\right)^{F}}^{\left(\boldsymbol{G}^{1}\right)^{F}} \pi_{x}^{\boldsymbol{G}^{1}}, f\right\rangle_{\left(\boldsymbol{T}^{1}\right)^{F}}=\left\langle\pi_{x}^{\boldsymbol{G}^{1}}, \operatorname{Ind}_{\boldsymbol{T}^{1}}^{\boldsymbol{G}^{1}} f\right\rangle_{\left(\boldsymbol{G}^{1}\right)^{F}}=\left|\left(\boldsymbol{T}^{0}\right)^{F}\right|^{-1} \sum_{\left\{g \in\left(\boldsymbol{G}^{0}\right)^{F} \mid g_{x} \in \boldsymbol{T}^{1}\right\}} f\left({ }^{g} x\right) .
$$

Using that $\left|\left(\boldsymbol{T}^{0}\right)^{F}\right|=\left|\left(\boldsymbol{T}^{1}\right)^{F}\right|$, we then get

$$
p^{\boldsymbol{G}^{1}}\left(\pi_{x}^{\boldsymbol{G}^{1}} \mathrm{St}_{\boldsymbol{G}^{1}}\right)=\varepsilon_{\boldsymbol{G}^{1}}\left|\left(\boldsymbol{G}^{1}\right)^{F}\right|^{-1} \sum_{\boldsymbol{T}^{1} \in \mathcal{T}_{1}^{F}} \sum_{\left\{g \in\left(\boldsymbol{G}^{0}\right)^{F} \mid g_{x} \in \boldsymbol{T}^{1}\right\}} \varepsilon_{\boldsymbol{T}^{1}} R_{\boldsymbol{T}^{1}}^{\boldsymbol{G}^{1}}\left(\pi_{g_{x}}^{\boldsymbol{T}^{1}}\right) .
$$

Taking ${ }^{g^{-1}} \boldsymbol{T}^{1}$ as summation index, we get

and hence

$$
p^{G^{1}}\left(\pi_{x}^{G^{1}} \operatorname{St}_{G^{1}}\right)=\varepsilon_{G^{1}} \sum_{\left\{T^{1} \in \mathcal{T}_{1}^{F} \mid T^{1} \ni x\right\}} \varepsilon_{T^{1}} R_{T^{1}}^{G^{1}}\left(\pi_{x}^{T^{1}}\right),
$$

$$
\pi_{x}^{\boldsymbol{G}^{1}}=\varepsilon_{\boldsymbol{G}^{x 0}}\left|\left(\boldsymbol{G}^{x 0}\right)^{F}\right|_{p}^{-1} \sum_{\left\{\boldsymbol{T}^{1} \in \mathcal{T}_{1}^{F} \mid \boldsymbol{T}^{1} \ni x\right\}} \varepsilon_{\boldsymbol{T}^{1}} R_{\boldsymbol{T}^{1}}^{\boldsymbol{G}^{1}}\left(\pi_{x}^{\boldsymbol{T}^{1}}\right),
$$

which is the first equality of the proposition.

For the second equality of the proposition, we first use [DM 1994, théorème 1.8(iii) and (iv)] to sum over tori of $C_{\boldsymbol{G}}(x)^{0}$ : the $\boldsymbol{T}^{1} \in \mathcal{T}_{1}^{F}$ containing $x$ are in bijection with the maximal tori of $C_{\boldsymbol{G}}(x)^{0}$ by $\boldsymbol{T}^{1} \mapsto\left(\boldsymbol{T}^{1^{x}}\right)^{0}$ and conversely $\boldsymbol{S} \mapsto C_{\boldsymbol{G}^{1}}(\boldsymbol{S})$. This bijection satisfies $\varepsilon_{\boldsymbol{T}^{1}}=\varepsilon_{S}$ by the definition of $\varepsilon$.

We then sum over $\left(C_{G}(x)^{0}\right)^{F}$-conjugacy classes of maximal tori, which are parameterized by $F$-conjugacy classes of $W^{0}(x)$. We then have to multiply by $\left|\left(C_{\boldsymbol{G}}(x)^{0}\right)^{F}\right| /\left|N_{\left(C_{\boldsymbol{G}}(x)^{0}\right)}(\boldsymbol{S})^{F}\right|$ the term indexed by the class of $\boldsymbol{S}$. Then we sum over the elements of $W^{0}(x)$. We then have to multiply the term indexed by $w$ by $\left|C_{W^{0}(x)}(w F)\right| /\left|W^{0}(x)\right|$. Using $\left|N_{\left(C_{G}(x)^{0}\right)}(S)^{F}\right|=\left|S^{F}\right|\left|C_{W^{0}(x)}(w F)\right|$, and the formula for $\operatorname{dim} R_{\boldsymbol{T}_{w}}^{C_{\boldsymbol{G}}(x)^{0}}$ (Id) we get the result. 


\section{Classification of quasi-semisimple classes}

The first items of this section, before Proposition 7.7, apply for algebraic groups over an arbitrary algebraically closed field $k$.

We denote by $\mathcal{C}\left(\boldsymbol{G}^{1}\right)$ the set of conjugacy classes of $\boldsymbol{G}^{1}$, that is, the orbits under $\boldsymbol{G}^{0}$-conjugacy, and denote by $\mathcal{C}\left(\boldsymbol{G}^{1}\right)_{\text {qss }}$ the set of quasi-semisimple classes.

Proposition 7.1. For $\boldsymbol{T}^{1} \in \mathcal{T}_{1}$, write $\boldsymbol{T}^{1}=\boldsymbol{T}^{0} \cdot \sigma$, where $\sigma$ is quasicentral. Then $\mathcal{C}\left(\boldsymbol{G}^{1}\right)_{\mathrm{qss}}$ is in bijection with the set of $N_{\boldsymbol{G}^{0}}\left(\boldsymbol{T}^{1}\right)$-orbits in $\boldsymbol{T}^{1}$, which itself is in bijection with the set of $W^{\sigma}$-orbits in $\mathcal{C}\left(\boldsymbol{T}^{1}\right)$, where $W=N_{\boldsymbol{G}^{0}}\left(\boldsymbol{T}^{0}\right) / \boldsymbol{T}^{0}$. We have $\mathcal{C}\left(\boldsymbol{T}^{1}\right) \simeq \boldsymbol{T}^{1} / \mathcal{L}_{\sigma}\left(\boldsymbol{T}^{0}\right)$, where $\mathcal{L}_{\sigma}$ is the map $t \mapsto t^{-1} \cdot{ }^{\sigma} t$.

Proof. By definition, every quasi-semisimple element of $\boldsymbol{G}^{1}$ is in some $\boldsymbol{T}^{1} \in \mathcal{T}_{1}$ and $\mathcal{T}_{1}$ is a single orbit under $\boldsymbol{G}^{0}$-conjugacy. It is thus sufficient to find how classes of $\boldsymbol{G}^{1}$ intersect $\boldsymbol{T}^{1}$. By [DM 1994, proposition 1.13], two elements of $\boldsymbol{T}^{1}$ are $\boldsymbol{G}^{0}$-conjugate if and only if they are conjugate under $N_{\boldsymbol{G}^{0}}\left(\boldsymbol{T}^{0}\right)$. We can replace $N_{\boldsymbol{G}^{0}}\left(\boldsymbol{T}^{0}\right)$ by $N_{\boldsymbol{G}^{0}}\left(\boldsymbol{T}^{1}\right)$ since if ${ }^{g}(\sigma t)=\sigma t^{\prime}$, where $g \in N_{\boldsymbol{G}^{0}}\left(\boldsymbol{T}^{0}\right)$, then the image of $g$ in $W$ lies in $W^{\sigma}$. By [DM 1994, définition-théorème 1.15(iii)], elements of $W^{\sigma}$ have representatives in $\boldsymbol{G}^{\sigma 0}$. Write $g=s \dot{w}$, where $\dot{w}$ is such a representative and $s \in \boldsymbol{T}^{0}$. Then ${ }^{s \dot{w}}(t \sigma)=\mathcal{L}_{\sigma}\left(s^{-1}\right)^{w} t \sigma$, whence the proposition.

\section{Lemma 7.2. $\quad \boldsymbol{T}^{0}=\boldsymbol{T}^{\sigma 0} \cdot \mathcal{L}_{\sigma}\left(\boldsymbol{T}^{0}\right)$.}

Proof. This is proved in [DM 1994, corollaire 1.33] when $\sigma$ is unipotent (and then the product is direct). We proceed similarly to that proof: $\boldsymbol{T}^{\sigma 0} \cap \mathcal{L}_{\sigma}\left(\boldsymbol{T}^{0}\right)$ is finite since its exponent divides the order of $\sigma$ (if $\sigma\left(t^{-1 \sigma} t\right)=t^{-1 \sigma} t$ then $\left(t^{-1 \sigma} t\right)^{n}=t^{-1 \sigma^{n}} t$ for all $n \geq 1)$, and $\operatorname{dim}\left(\boldsymbol{T}^{\sigma 0}\right)+\operatorname{dim}\left(\mathcal{L}_{\sigma}\left(\boldsymbol{T}^{0}\right)\right)=\operatorname{dim}\left(\boldsymbol{T}^{0}\right)$ as the exact sequence $1 \rightarrow \boldsymbol{T}^{0^{\sigma}} \rightarrow \boldsymbol{T}^{0} \rightarrow \mathcal{L}_{\sigma}\left(\boldsymbol{T}^{0}\right) \rightarrow 1$ shows, using that $\operatorname{dim}\left(\boldsymbol{T}^{\sigma 0}\right)=\operatorname{dim} \boldsymbol{T}^{0^{\sigma}}$.

It follows that $\boldsymbol{T}^{0} / \mathcal{L}_{\sigma}\left(\boldsymbol{T}^{0}\right) \simeq \boldsymbol{T}^{\sigma 0} /\left(\boldsymbol{T}^{\sigma 0} \cap \mathcal{L}_{\sigma}\left(\boldsymbol{T}^{0}\right)\right)$; since the set $\mathcal{C}\left(\boldsymbol{G}^{\sigma 0}\right)_{\mathrm{ss}}$ of semisimple classes of $\boldsymbol{G}^{\sigma 0}$ identifies with the set of $W^{\sigma}$-orbits on $\boldsymbol{T}^{\sigma 0}$, this induces a surjective map $\mathcal{C}\left(\boldsymbol{G}^{\sigma 0}\right)_{\mathrm{ss}} \rightarrow \mathcal{C}\left(\boldsymbol{G}^{1}\right)_{\text {qss }}$.

Example 7.3. We will describe the quasi-semisimple classes of $\boldsymbol{G}^{0} \cdot \sigma$, where $\boldsymbol{G}^{0}=\mathrm{GL}_{n}(k)$ and $\sigma$ is the quasicentral automorphism given by $\sigma(g)=J^{t} g^{-1} J^{-1}$, where, if $n$ is even, $J$ is the matrix $\left(\begin{array}{cc}0 & -J_{0} \\ J_{0} & 0\end{array}\right)$ with

$$
J_{0}=\left(\begin{array}{lll}
0 & & 1 \\
& . & \\
1 & & 0
\end{array}\right),
$$

and if $n$ is odd, $J$ is the antidiagonal matrix

$$
J=\left(\begin{array}{lll}
0 & & 1 \\
& \cdot \cdot & \\
1 & & 0
\end{array}\right)
$$


(any outer algebraic automorphism of $\mathrm{GL}_{n}$ is equal to $\sigma$ up to an inner automorphism).

The automorphism $\sigma$ normalizes the pair $\boldsymbol{T}^{0} \subset \boldsymbol{B}^{0}$, where $\boldsymbol{T}^{0}$ is the diagonal torus and $\boldsymbol{B}^{0}$ the group of upper triangular matrices. Then $\boldsymbol{T}^{1}=N_{\boldsymbol{G}^{1}}\left(\boldsymbol{T}^{0} \subset \boldsymbol{B}^{0}\right)=\boldsymbol{T}^{0} \cdot \sigma$ is in $\mathcal{T}_{1}$. For $\operatorname{diag}\left(x_{1}, \ldots, x_{n}\right) \in \boldsymbol{T}^{0}$, where $x_{i} \in k^{\times}$, we have $\sigma\left(\operatorname{diag}\left(x_{1}, \ldots, x_{n}\right)\right)=$ $\operatorname{diag}\left(x_{n}^{-1}, \ldots, x_{1}^{-1}\right)$. It follows that $\mathcal{L}_{\sigma}\left(\boldsymbol{T}^{0}\right)=\left\{\operatorname{diag}\left(x_{1}, x_{2}, \ldots, x_{2}, x_{1}\right)\right\}-$ here $x_{m+1}$ is a square when $n=2 m+1$ but this is not a condition since $k$ is algebraically closed. As suggested above, we could take as representatives of $\boldsymbol{T}^{0} / \mathcal{L}_{\sigma}\left(\boldsymbol{T}^{0}\right)$ the set $\boldsymbol{T}^{\sigma 0} /\left(\boldsymbol{T}^{\sigma 0} \cap \mathcal{L}_{\sigma}\left(\boldsymbol{T}^{0}\right)\right)$, but since $\boldsymbol{T}^{\sigma 0} \cap \mathcal{L}_{\sigma}\left(\boldsymbol{T}^{0}\right)$ is not trivial (it consists of the diagonal matrices with entries \pm 1 placed symmetrically), it is more convenient to take for representatives of the quasi-semisimple classes, the set $\left\{\operatorname{diag}\left(x_{1}, x_{2}, \ldots, x_{\lfloor n / 2\rfloor}, 1, \ldots, 1\right)\right\} \sigma$. In this model, the action of $W^{\sigma}$ is generated by the permutations of the $\lfloor n / 2\rfloor$ first entries, and by the maps $x_{i} \mapsto x_{i}^{-1}$, so the quasi-semisimple classes of $\boldsymbol{G}^{0} \cdot \sigma$ are parameterized by the quasi-semisimple classes of $\boldsymbol{G}^{\sigma 0}$.

We continue the example, computing group of components of centralizers.

Proposition 7.4. Let $s \sigma=\operatorname{diag}\left(x_{1}, x_{2}, \ldots, x_{\lfloor n / 2\rfloor}, 1, \ldots, 1\right) \sigma$ be a quasi-semisimple element as above. If char $k=2$ then $C_{G^{0}}(s \sigma)$ is connected. Otherwise, if $n$ is odd, $A(s \sigma):=C_{G^{0}}(s \sigma) / C_{G^{0}}(s \sigma)^{0}$ is of order two, generated by $-1 \in$ $Z G^{0}=Z G_{n}(k)$. If $n$ is even, $A(s \sigma) \neq 1$ if and only if for some $i$, we have $x_{i}=-1$; then $x_{i} \mapsto x_{i}^{-1}$ is an element of $W^{\sigma}$ which has a representative in $C_{\boldsymbol{G}^{0}}(s \sigma)$ generating $A(s \sigma)$, which is of order 2 .

Proof. We will use that for a group $G$ and an automorphism $\sigma$ of $G$, we have an exact sequence (see, for example, [Steinberg 1968, 4.5])

$$
1 \rightarrow(Z G)^{\sigma} \rightarrow G^{\sigma} \rightarrow(G / Z G)^{\sigma} \rightarrow\left(\mathcal{L}_{\sigma}(G) \cap Z G\right) / \mathcal{L}_{\sigma}(Z G) \rightarrow 1 .
$$

If we take $G=\boldsymbol{G}^{0}=\mathrm{GL}_{n}(k)$ in (7.5) and $s \sigma$ for $\sigma$, since on $Z \boldsymbol{G}^{0}$ the map $\mathcal{L}_{\sigma}=\mathcal{L}_{s \sigma}$ is $z \mapsto z^{2}$, hence surjective, we get that $\boldsymbol{G}^{0^{s \sigma}} \rightarrow \mathrm{PGL}_{n}^{s \sigma}$ is surjective and has kernel $\left(Z \boldsymbol{G}^{0}\right)^{\sigma}=\{ \pm 1\}$.

Assume $n$ odd and take $G=\mathrm{SL}_{n}(k)$ in (7.5). We have $Z \mathrm{SL}_{n}^{\sigma}=\{1\}$ so that we get the following diagram with exact rows:

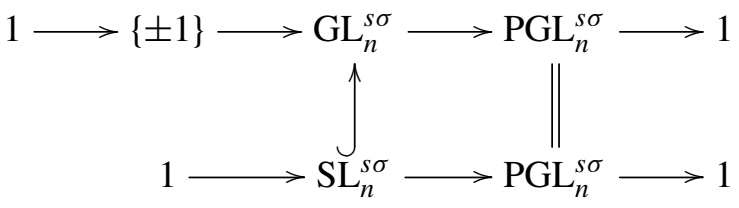

This shows that $\mathrm{GL}_{n}^{s \sigma} / \mathrm{SL}_{n}^{s \sigma} \simeq\{ \pm 1\}$; by [Steinberg 1968, 8.1], $\mathrm{SL}_{n}^{s \sigma}$ is connected, hence $\mathrm{PGL}_{n}^{s \sigma}$ is connected. Thus $\mathrm{GL}_{n}^{s \sigma}=\left(\mathrm{GL}_{n}^{s \sigma}\right)^{0} \times\{ \pm 1\}$ is connected if and only if $\operatorname{char} k=2$. 
Assume now that $n$ is even; then $\left(\boldsymbol{T}^{0}\right)^{\sigma}$ is connected, and hence $-1 \in\left(\mathrm{GL}_{n}^{s \sigma}\right)^{0}$ for all $s \in \boldsymbol{T}^{0}$. Using this, the exact sequence $1 \rightarrow\{ \pm 1\} \rightarrow \mathrm{GL}_{n}^{s \sigma} \rightarrow \mathrm{PGL}_{n}^{s \sigma} \rightarrow 1$ implies $A(s \sigma)=\boldsymbol{G}^{s \sigma} / \boldsymbol{G}^{0 s \sigma}=\mathrm{GL}_{n}^{s \sigma} /\left(\mathrm{GL}_{n}^{s \sigma}\right)^{0} \simeq \mathrm{PGL}_{n}^{s \sigma} /\left(\mathrm{PGL}_{n}^{s \sigma}\right)^{0}$. To compute this group we use (7.5) with $\mathrm{SL}_{n}(k)$ for $G$ and $s \sigma$ for $\sigma$ :

$$
1 \rightarrow\{ \pm 1\} \rightarrow \mathrm{SL}_{n}^{s \sigma} \rightarrow \mathrm{PGL}_{n}^{s \sigma} \rightarrow\left(\mathcal{L}_{s \sigma}\left(\mathrm{SL}_{n}\right) \cap Z \mathrm{SL}_{n}\right) / \mathcal{L}_{\sigma}\left(Z \mathrm{SL}_{n}\right) \rightarrow 1
$$

which, since $\mathrm{SL}_{n}^{s \sigma}$ is connected, implies that

$$
A(s \sigma)=\left(\mathcal{L}_{s \sigma}\left(\mathrm{SL}_{n}\right) \cap Z \mathrm{SL}_{n}\right) / \mathcal{L}_{\sigma}\left(Z \mathrm{SL}_{n}\right)
$$

is nontrivial (of order 2) if and only if $\mathcal{L}_{s \sigma}\left(\mathrm{SL}_{n}\right) \cap Z \mathrm{SL}_{n}$ contains an element which is not a square in $Z \mathrm{SL}_{n}$; thus $A(s \sigma)$ is trivial if char $k=2$. We assume now that char $k \neq 2$. Then a nonsquare is of the form $\operatorname{diag}(z, \ldots, z)$ with $z^{m}=-1$ if we set $m=n / 2$.

The following lemma is a transcription of [Steinberg 1968, 9.5].

Lemma 7.6. Let $\sigma$ be a quasicentral automorphism of the connected reductive group $\boldsymbol{G}$ which stabilizes the pair $\boldsymbol{T} \subset \boldsymbol{B}$ of a maximal torus and a Borel subgroup; let $W$ be the Weyl group of $\boldsymbol{T}$ and let $s \in \boldsymbol{T}$. Then

$$
\boldsymbol{T} \cap \mathcal{L}_{s \sigma}(\boldsymbol{G})=\left\{\mathcal{L}_{w}\left(s^{-1}\right) \mid w \in W^{\sigma}\right\} \cdot \mathcal{L}_{\sigma}(\boldsymbol{T}) .
$$

Proof. Assume $t=\mathcal{L}_{s \sigma}(x)$ for $t \in \boldsymbol{T}$, or equivalently $x t={ }^{s \sigma} x$. Then if $x$ is in the Bruhat cell $\boldsymbol{B} w \boldsymbol{B}$, we must have $w \in W^{\sigma}$. Taking for $w$ a $\sigma$-stable representative $\dot{w}$ and writing the unique Bruhat decomposition $x=u_{1} \dot{w} t_{1} u_{2}$, where $u_{2} \in \boldsymbol{U}, t_{1} \in \boldsymbol{T}$ and $u_{1} \in \boldsymbol{U} \cap{ }^{w} \boldsymbol{U}^{-}$, where $\boldsymbol{U}$ is the unipotent radical of $\boldsymbol{B}$ and $\boldsymbol{U}^{-}$the unipotent radical of the opposite Borel, the equality $x t={ }^{s \sigma} x$ implies that $\dot{w} t_{1} t={ }^{s \sigma}\left(\dot{w} t_{1}\right)$, or equivalently, $t=\mathcal{L}_{w^{-1}}\left(s^{-1}\right) \mathcal{L}_{\sigma}\left(t_{1}\right)$, whence the lemma.

We apply this lemma taking $\mathrm{SL}_{n}$ for $\boldsymbol{G}$ and $\boldsymbol{T}^{\prime 0}=\boldsymbol{T}^{0} \cap \mathrm{SL}_{n}$ for $\boldsymbol{T}$ : we get $\mathcal{L}_{s \sigma}\left(\mathrm{SL}_{n}\right) \cap Z \mathrm{SL}_{n}=\left\{\mathcal{L}_{w}\left(s^{-1}\right) \mid w \in W^{\sigma}\right\} \cdot \mathcal{L}_{\sigma}\left(\boldsymbol{T}^{\prime 0}\right) \cap Z \mathrm{SL}_{n}$. The element

$$
\operatorname{diag}\left(x_{1}, x_{2}, \ldots, x_{m}, 1, \ldots, 1\right) \sigma
$$

is conjugate to

$$
s \sigma=\operatorname{diag}\left(y_{1}, y_{2}, \ldots, y_{m}, y_{m}^{-1}, \ldots, y_{1}^{-1}\right) \sigma \in\left(\boldsymbol{T}^{\prime 0}\right)^{\sigma} \cdot \sigma,
$$

where $y_{i}^{2}=x_{i}$. It will have a nonconnected centralizer if and only if for some $w \in W^{\sigma}$ and some $t \in \boldsymbol{T}^{\prime 0}$, we have $\mathcal{L}_{w}\left(s^{-1}\right) \cdot \mathcal{L}_{\sigma}(t)=\operatorname{diag}(z, \ldots, z)$ with $z^{m}=-1$, and then an appropriate representative of $w$ (multiplying if needed by an element of $Z \mathrm{GL}_{n}$ ) will be in $C_{G^{0}}(s \sigma)$ and have a nontrivial image in $A(s \sigma)$. Since $s$ and $w$ are $\sigma$-fixed, we have $\mathcal{L}_{w}(s) \in\left(\boldsymbol{T}^{\prime 0}\right)^{\sigma}$; thus it is of the form $\operatorname{diag}\left(a_{1}, \ldots, a_{m}, a_{m}^{-1}, \ldots a_{1}^{-1}\right)$. Since

$$
\mathcal{L}_{\sigma}\left(\boldsymbol{T}^{\prime 0}\right)=\left\{\operatorname{diag}\left(t_{1}, \ldots, t_{m}, t_{m}, \ldots, t_{1}\right) \mid t_{1} t_{2} \cdots t_{m}=1\right\},
$$


we get $z=a_{1} t_{1}=a_{2} t_{2}=\cdots=a_{m} t_{m}=a_{m}^{-1} t_{m}=\cdots=a_{1}^{-1} t_{1}$; in particular, $a_{i}= \pm 1$ for all $i$ and $a_{1} a_{2} \cdots a_{m}=-1$. We can take $w$ up to conjugacy in $W^{\sigma}$ since $\mathcal{L}_{v w v^{-1}}\left(s^{-1}\right)={ }^{v} \mathcal{L}_{w}\left(v^{-1} s^{-1}\right)$ and $\mathcal{L}_{\sigma}\left(\boldsymbol{T}^{\prime 0}\right)$ is invariant under $W^{\sigma}$-conjugacy. We see $W^{\sigma}$ as the group of permutations of $\{1,2, \ldots, m,-m, \ldots,-1\}$ which preserves the pairs $\{i,-i\}$. A nontrivial cycle of $w$ has, up to conjugacy, either the form $(1,-1)$ or $\left(1,-2,3, \ldots,(-1)^{i-1} i,-(i+1),-(i+2), \ldots,-k,-1,2,-3, \ldots, k\right)$ with $0 \leq i \leq k \leq n$ and $i$ odd, or $\left(1,-2,3, \ldots,(-1)^{i-1} i, i+1, i+2, \ldots, k\right)$ with $0 \leq i \leq k \leq n$ and $i$ even (the case $i=0$ meaning that there is no sign change). The contribution to $a_{1} \cdots a_{m}$ of the orbit $(1,-1)$ is $a_{1}=y_{1}^{2}$, and hence is 1 except if $y_{1}^{2}=x_{1}=-1$. Let us consider an orbit of the second form. The $k$ first coordinates of $\mathcal{L}_{w}\left(s^{-1}\right)$ are $\left(y_{1} y_{2}, \ldots, y_{i} y_{i+1}, y_{i+1} / y_{i+2}, \ldots, y_{k} / y_{1}\right)$. Hence there must exist signs $\varepsilon_{j}$ such that $y_{2}=\varepsilon_{1} / y_{1}, y_{3}=\varepsilon_{2} / y_{2}, \ldots, y_{i+1}=\varepsilon_{i} / y_{i}$ and $y_{i+2}=\varepsilon_{i+1} y_{i+1}, \ldots, y_{k}=\varepsilon_{k-1} y_{k-1}, y_{1}=\varepsilon_{k} y_{k}$. This gives

$$
y_{1}= \begin{cases}\varepsilon_{1} \cdots \varepsilon_{k} y_{1} & \text { if } i \text { is even, } \\ \varepsilon_{1} \cdots \varepsilon_{k} / y_{1} & \text { if } i \text { is odd. }\end{cases}
$$

The contribution of the orbit to $a_{1} \cdots a_{m}$ is $\varepsilon_{1} \cdots \varepsilon_{k}$ and thus is 1 if $i$ is even and $x_{1}=y_{1}^{2}$ if $i$ is odd. Again, we see that one of the $x_{i}$ must equal -1 to get $a_{1} \cdots a_{m}=-1$. Conversely if $x_{1}=-1$, for any $z$ such that $z^{m}=-1$, choosing $t$ such that $\mathcal{L}_{\sigma}(t)=\operatorname{diag}(-z, z, z, \ldots, z,-z)$ and taking $w=(1,-1)$, we get $\mathcal{L}_{w}\left(s^{-1}\right) \mathcal{L}_{\sigma}(t)=\operatorname{diag}(z, \ldots, z)$ as desired.

We now go back to the case where $k=\overline{\mathbb{F}}_{q}$, and in the context of Proposition 7.1, we now assume that $\boldsymbol{T}^{1}$ is $F$-stable and that $\sigma$ induces an $F$-stable automorphism of $\boldsymbol{G}^{0}$.

Proposition 7.7. Let $\boldsymbol{T}^{1 \mathrm{rat}}=\left\{s \in \boldsymbol{T}^{1} \mid \exists n \in N_{\boldsymbol{G}^{0}}\left(\boldsymbol{T}^{1}\right),{ }^{n F} s=s\right\}$; then $\boldsymbol{T}^{1 \mathrm{rat}}$ is stable by $\boldsymbol{T}^{0}$-conjugacy, which gives a meaning to $\mathcal{C}\left(\boldsymbol{T}^{1 \text { rat }}\right)$. Then $c \mapsto c \cap \boldsymbol{T}^{1}$ induces a bijection between $\left(\mathcal{C}\left(\boldsymbol{G}^{1}\right)_{\mathrm{qss}}\right)^{F}$ and the $W^{\sigma}$-orbits on $\mathcal{C}\left(\boldsymbol{T}^{1 \text { rat }}\right)$.

Proof. A class $c \in \mathcal{C}\left(\boldsymbol{G}^{1}\right)_{\mathrm{qss}}$ is $F$-stable if and only if given $s \in c$, we have ${ }^{F} \in c$. If we take $s \in c \cap \boldsymbol{T}^{1}$ then ${ }^{F_{S}} \in c \cap \boldsymbol{T}^{1}$, which as observed in the proof of Proposition 7.1 implies that ${ }^{F} s$ is conjugate to $s$ under $N_{\boldsymbol{G}^{0}}\left(\boldsymbol{T}^{1}\right)$, that is, $s \in \boldsymbol{T}^{1 \text { rat }}$. Thus $c$ is $F$-stable if and only if $c \cap \boldsymbol{T}^{1}=c \cap \boldsymbol{T}^{1 \text { rat }}$. The proposition then results from Proposition 7.1, observing that $\boldsymbol{T}^{1 \text { rat }}$ is stable under $N_{\boldsymbol{G}^{0}}\left(\boldsymbol{T}^{1}\right)$-conjugacy and that the corresponding orbits are the $W^{\sigma}$-orbits on $\mathcal{C}\left(\boldsymbol{T}^{1 \text { rat }}\right)$.

Example 7.8. When $\boldsymbol{G}^{1}=\mathrm{GL}_{n}\left(\overline{\mathbb{F}}_{q}\right) \cdot \sigma$ with $\sigma$ as in Example 7.3, the map $\operatorname{diag}\left(x_{1}, x_{2}, \ldots, x_{\lfloor n / 2\rfloor}, 1, \ldots, 1\right) \mapsto \operatorname{diag}\left(x_{1}, x_{2}, \ldots, x_{\lfloor n / 2\rfloor}, \dagger, x_{\lfloor n / 2\rfloor}^{-1}, \ldots, x_{2}^{-1}, x_{1}^{-1}\right)$, where $\dagger$ represents 1 if $n$ is odd and an omitted entry otherwise, is compatible with the action of $W^{\sigma}$ as described in Example 7.3 on the left-hand side and the natural action on the right-hand side. This map induces a bijection from $\mathcal{C}\left(\boldsymbol{G}^{1}\right)_{\mathrm{qss}}$ to the 
semisimple classes of $\left(\mathrm{GL}_{n}^{\sigma}\right)^{0}$ which restricts to a bijection from $\left(\mathcal{C}\left(\boldsymbol{G}^{1}\right)_{\mathrm{qss}}\right)^{F}$ to the $F$-stable semisimple classes of $\left(\mathrm{GL}_{n}^{\sigma}\right)^{0}$.

We now compute the cardinality of $\left(\mathcal{C}\left(\boldsymbol{G}^{1}\right)_{\mathrm{qss}}\right)^{F}$.

Proposition 7.9. Let $f$ be a function on $\left(\mathcal{C}\left(G^{1}\right)_{\mathrm{qss}}\right)^{F}$. Then

$$
\sum_{c \in\left(\mathcal{C}\left(\boldsymbol{G}^{1}\right)_{\mathrm{qss}}\right)^{F}} f(c)=\left|W^{\sigma}\right|^{-1} \sum_{w \in W^{\sigma}} \tilde{f}(w)
$$

where $\tilde{f}(w):=\sum_{s} f(s)$, where s runs over representatives of $\boldsymbol{T}^{1 w F} / \mathcal{L}_{\sigma}\left(\boldsymbol{T}^{0}\right)^{w F}$ in $\boldsymbol{T}^{1^{w F}}$.

Proof. We have

$$
\mathcal{C}\left(\boldsymbol{T}^{1 \mathrm{rat}}\right)=\bigcup_{w \in W^{\sigma}}\left\{s \mathcal{L}_{\sigma}\left(\boldsymbol{T}^{0}\right) \in \boldsymbol{T}^{1} / \mathcal{L}_{\sigma}\left(\boldsymbol{T}^{0}\right) \mid s \mathcal{L}_{\sigma}\left(\boldsymbol{T}^{0}\right) \text { is } w F \text {-stable }\right\} .
$$

The conjugation by $v \in W^{\sigma}$ sends a $w F$-stable coset $s \mathcal{L}_{\sigma}\left(\boldsymbol{T}^{0}\right)$ to a $v w F v^{-1}$ stable coset; and the number of $w$ such that $s \mathcal{L}_{\sigma}\left(\boldsymbol{T}^{0}\right)$ is $w F$-stable is equal to $N_{W^{\sigma}}\left(s \mathcal{L}_{\sigma}\left(\boldsymbol{T}^{0}\right)\right)$. It follows that

$$
\sum_{c \in\left(\mathcal{C}\left(\boldsymbol{G}^{1}\right)_{\mathrm{qss}}\right)^{F}} f(c)=\left|W^{\sigma}\right|^{-1} \sum_{w \in W^{\sigma}} \sum_{s \mathcal{L}_{\sigma}\left(\boldsymbol{T}^{0}\right) \in\left(\boldsymbol{T}^{1} / \mathcal{L}_{\sigma}\left(\boldsymbol{T}^{0}\right)\right)^{w F}} f\left(s \mathcal{L}_{\sigma}\left(\boldsymbol{T}^{0}\right)\right) .
$$

The proposition follows since, because $\mathcal{L}_{\sigma}\left(\boldsymbol{T}^{0}\right)$ is connected, we have

$$
\left(\boldsymbol{T}^{1} / \mathcal{L}_{\sigma}\left(\boldsymbol{T}^{0}\right)\right)^{w F}=\boldsymbol{T}^{1^{w F}} / \mathcal{L}_{\sigma}\left(\boldsymbol{T}^{0}\right)^{w F} .
$$

Corollary 7.10. We have $\left|\left(\mathcal{C}\left(\boldsymbol{G}^{1}\right)_{\mathrm{qss}}\right)^{F}\right|=\left|\left(\mathcal{C}\left(\boldsymbol{G}^{\sigma 0}\right)_{s s}\right)^{F}\right|$.

Proof. Let us take $f=1$ in Proposition 7.9. We need to sum over $w \in W^{\sigma}$ the value $\left|\boldsymbol{T}^{1^{w F}} / \mathcal{L}_{\sigma}\left(\boldsymbol{T}^{0}\right)^{w F}\right|$. First note that $\left|\boldsymbol{T}^{1^{w F}} / \mathcal{L}_{\sigma}\left(\boldsymbol{T}^{0}\right)^{w F}\right|=\left|\boldsymbol{T}^{0^{w F}} / \mathcal{L}_{\sigma}\left(\boldsymbol{T}^{0}\right)^{w F}\right|$. By Lemma 7.2 we have the exact sequence

$$
1 \rightarrow \boldsymbol{T}^{\sigma 0} \cap \mathcal{L}_{\sigma}\left(\boldsymbol{T}^{0}\right) \rightarrow \boldsymbol{T}^{\sigma 0} \times \mathcal{L}_{\sigma}\left(\boldsymbol{T}^{0}\right) \rightarrow \boldsymbol{T}^{0} \rightarrow 1,
$$

whence the Galois cohomology exact sequence is

$$
\begin{aligned}
1 \rightarrow\left(\boldsymbol{T}^{\sigma 0} \cap \mathcal{L}_{\sigma}\left(\boldsymbol{T}^{0}\right)\right)^{w F} \rightarrow \boldsymbol{T}^{\sigma 0^{w F}} \times & \left(\mathcal{L}_{\sigma}\left(\boldsymbol{T}^{0}\right)\right)^{w F} \\
& \rightarrow \boldsymbol{T}^{0 w F} \rightarrow H^{1}\left(w F,\left(\boldsymbol{T}^{\sigma 0} \cap \mathcal{L}_{\sigma}\left(\boldsymbol{T}^{0}\right)\right)\right) \rightarrow 1 .
\end{aligned}
$$

Using that for any automorphism $\tau$ of a finite group $G$ we have $\left|G^{\tau}\right|=\left|H^{1}(\tau, G)\right|$, we have

$$
\left|\left(\boldsymbol{T}^{\sigma 0} \cap \mathcal{L}_{\sigma}\left(\boldsymbol{T}^{0}\right)\right)^{w F}\right|=\left|H^{1}\left(w F,\left(\boldsymbol{T}^{\sigma 0} \cap \mathcal{L}_{\sigma}\left(\boldsymbol{T}^{0}\right)\right)\right)\right| .
$$


Together with the above exact sequence, this implies that $\left|\boldsymbol{T}^{0^{w F}} / \mathcal{L}_{\sigma}\left(\boldsymbol{T}^{0}\right)^{w F}\right|=$ $\left|\boldsymbol{T}^{\sigma 0^{w F}}\right|$, whence

$$
\left|\left(\mathcal{C}\left(\boldsymbol{G}^{1}\right)_{\mathrm{qss}}\right)^{F}\right|=\left|W^{\sigma}\right|^{-1} \sum_{w \in W^{\sigma}}\left|\boldsymbol{T}^{\sigma 0^{w F}}\right| .
$$

The corollary follows by either applying the same formula for the connected group $\boldsymbol{G}^{\sigma 0}$, or referring to [Lehrer 1992, Proposition 2.1].

\section{Shintani descent}

We now look at Shintani descent in our context; we will show it commutes with Lusztig induction when $\boldsymbol{G}^{1} / \boldsymbol{G}^{0}$ is semisimple and the characteristic is good for $\boldsymbol{G}^{\sigma 0}$. We should mention previous work on this subject: Eftekhari [1996, II.3.4] has the same result for Lusztig induction from a torus; he does not need to assume $p$ good but needs $q$ to be large enough to apply results of Lusztig, identifying DeligneLusztig induction with induction of character sheaves; Digne [1999, 1.1] has the result in the same generality as here apart from the assumption that $G^{1}$ contains an $F$-stable quasicentral element; however, a defect of his proof is the use without proof of the property given in Lemma 8.4 below.

As above, $\boldsymbol{G}^{1}$ denotes an $F$-stable connected component of $\boldsymbol{G}$ of the form $\boldsymbol{G}^{0} \cdot \sigma$, where $\sigma$ induces a quasicentral automorphism of $\boldsymbol{G}^{0}$ commuting with $F$.

Applying Lang's theorem, one can write any element of $\boldsymbol{G}^{1}$ as $x \cdot{ }^{\sigma F} x^{-1} \sigma$ for some $x \in \boldsymbol{G}^{0}$, or as $\sigma \cdot{ }^{F} x^{-1} \cdot x$ for some $x \in \boldsymbol{G}^{0}$. Using that $\sigma$, as an automorphism, commutes with $F$, it is easy to check that the correspondence $x \cdot{ }^{\sigma F} x^{-1} \sigma \mapsto \sigma^{F} x^{-1} \cdot x$ induces a bijection $n_{F / \sigma F}$ from the $\left(\boldsymbol{G}^{0}\right)^{F}$-conjugacy classes of $\left(\boldsymbol{G}^{1}\right)^{F}$ to the $\boldsymbol{G}^{0^{\sigma F}}$ conjugacy classes of $\left(\boldsymbol{G}^{1}\right)^{\sigma F}$ and that $\left|\boldsymbol{G}^{0^{\sigma F}}\right||c|=\left|\left(\boldsymbol{G}^{0}\right)^{F}\right|\left|n_{F / \sigma F}(c)\right|$ for any $\left(\boldsymbol{G}^{0}\right)^{F}$ class $c$ in $\left(\boldsymbol{G}^{1}\right)^{F}$. It follows that the operator $\operatorname{sh}_{F / \sigma F}$ from $\left(\boldsymbol{G}^{0}\right)^{F}$-class functions on $\left(\boldsymbol{G}^{1}\right)^{F}$ to $\boldsymbol{G}^{0^{\sigma F}}$-class functions on $\left(\boldsymbol{G}^{1}\right)^{\sigma F}$ defined by $\operatorname{sh}_{F / \sigma F}(\chi)\left(n_{F / \sigma F} x\right)=\chi(x)$ is an isometry.

The remainder of this section is devoted to the proof of the following:

Proposition 8.1. Let $\boldsymbol{L}^{1}=N_{\boldsymbol{G}^{1}}\left(\boldsymbol{L}^{0} \subset \boldsymbol{P}^{0}\right)$ be a Levi of $\boldsymbol{G}^{1}$ containing $\sigma$, where $\boldsymbol{L}^{0}$ is $F$-stable; we have $\boldsymbol{L}^{1}=\boldsymbol{L}^{0} \cdot \sigma$. Assume that $\sigma$ is semisimple and that the characteristic is good for $\boldsymbol{G}^{\sigma 0}$. Then

$$
\operatorname{sh}_{F / \sigma F} \circ{ }^{*} R_{L^{1}}^{G^{1}}={ }^{*} R_{L^{1}}^{G^{1}} \circ \operatorname{sh}_{F / \sigma F} \quad \text { and } \quad \operatorname{sh}_{F / \sigma F} \circ R_{L^{1}}^{G^{1}}=R_{L^{1}}^{G^{1}} \circ \operatorname{sh}_{F / \sigma F} .
$$

Proof. The second equality follows from the first by adjunction, using that the adjoint of $\operatorname{sh}_{F / \sigma F}$ is $\operatorname{sh}_{F / \sigma F}^{-1}$. Let us prove the first equality.

Let $\chi$ be a $\left(\boldsymbol{G}^{0}\right)^{F}$-class function on $\boldsymbol{G}^{1}$ and let $\sigma l u=u \sigma l$ be the Jordan decomposition of an element of $\left(\boldsymbol{L}^{1}\right)^{\sigma F}$ with $u$ unipotent and $\sigma l$ semisimple. By the character formula Proposition 2.5(iii) and the definition of $Q_{\boldsymbol{L}^{t 0}}^{\boldsymbol{G}^{t 0}}$ for $t=\sigma l$, we have 
$\left({ }^{*} R_{\boldsymbol{L}^{1}}^{\boldsymbol{G}^{1}} \operatorname{sh}_{F / \sigma F}(\chi)\right)(\sigma l u)$

$$
=\left|\left(\boldsymbol{G}^{\sigma l}\right)^{0^{\sigma F}}\right|^{-1} \sum_{v \in\left(\boldsymbol{G}^{\sigma l}\right)^{0_{\text {unip }}^{\sigma F}}} \operatorname{sh}_{F / \sigma F}(\chi)(\sigma l v) \operatorname{Trace}\left(\left(v, u^{-1}\right) \mid H_{c}^{*}\left(Y_{\boldsymbol{U}, \sigma F}\right)\right),
$$

where $v$ (resp. $u$ ) acts by left- (resp. right-) translation on

$$
Y_{\boldsymbol{U}, \sigma F}=\left\{x \in\left(\boldsymbol{G}^{\sigma l}\right)^{0} \mid x^{-1} \cdot{ }^{\sigma F} x \in \boldsymbol{U}\right\},
$$

where $\boldsymbol{U}$ denotes the unipotent radical of $\boldsymbol{P}^{0}$; in the summation, $v$ is in the identity component of $\boldsymbol{G}^{\sigma l}$ since, $\sigma$ being semisimple, $u$ is in $\boldsymbol{G}^{0}$ and hence in $\left(\boldsymbol{G}^{\sigma l}\right)^{0}$ by [DM 1994, théorème 1.8(i)] since $\sigma l$ is semisimple.

Let us write $l={ }^{F} \lambda^{-1} \cdot \lambda$ with $\lambda \in \boldsymbol{L}^{0}$, so that $\sigma l=n_{F / \sigma F}\left(l^{\prime} \sigma\right)$, where $l^{\prime}=\lambda \cdot \sigma F \lambda^{-1}$. Lemma 8.2. For $v \in\left(G^{\sigma l}\right)^{0^{\sigma F}} \underset{\text { unip }}{\text {, we have }}$

$$
\sigma l v=n_{F / \sigma F}\left(\left(\sigma l \cdot v^{\prime}\right)^{\sigma^{F} \lambda^{-1}}\right),
$$

where $v^{\prime}=n_{\sigma F / \sigma F} v \in\left(\boldsymbol{G}^{\sigma l}\right)^{0^{\sigma F}}$ is defined by writing $v={ }^{\sigma F} \eta \cdot \eta^{-1}$, where $\eta \in$ $\left(\boldsymbol{G}^{\sigma t}\right)^{0}$ and setting $v^{\prime}=\eta^{-1} \cdot{ }^{\sigma F} \eta$.

Proof. We have

$$
\sigma l v=\sigma l^{\sigma F} \eta \cdot \eta^{-1}={ }^{\sigma F} \eta \sigma l \eta^{-1}=\sigma^{F}\left(\eta \lambda^{-1}\right) \lambda \eta^{-1} ;
$$

thus $\sigma l v=n_{F / \sigma F}\left(\left(\lambda \eta^{-1}\right) \cdot \sigma F\left(\eta \lambda^{-1}\right) \sigma\right)$. And we have

$$
\left(\lambda \eta^{-1}\right) \cdot{ }^{\sigma F}\left(\eta \lambda^{-1}\right) \sigma=\lambda v^{\prime \sigma F} \lambda^{-1} \sigma={ }^{F} \lambda l v^{\prime} \sigma^{F} \lambda^{-1}=\left(\sigma l v^{\prime}\right)^{\sigma^{F} \lambda^{-1}} ;
$$

thus $\operatorname{sh}_{F / \sigma F}(\chi)(\sigma l v)=\chi\left(\left(\sigma l v^{\prime}\right)^{\sigma^{F} \lambda^{-1}}\right)$.

Lemma 8.3. (i) We have $(\sigma l)^{\sigma^{F} \lambda^{-1}}=l^{\prime} \sigma$.

(ii) The conjugation $x \mapsto x^{\sigma^{F} \lambda^{-1}}$ maps $\boldsymbol{G}^{\sigma l}$ and the action of $\sigma F$ on it to $\boldsymbol{G}^{l^{\prime} \sigma}$ with the action of $F$ on it; in particular, it induces bijections

$$
\left(\boldsymbol{G}^{\sigma l}\right)^{0^{\sigma F}} \stackrel{\sim}{\longrightarrow}\left(\boldsymbol{G}^{l^{\prime} \sigma}\right)^{0^{F}} \text { and } Y_{\boldsymbol{U}, \sigma F} \stackrel{\sim}{\longrightarrow} Y_{\boldsymbol{U}, F},
$$

where $Y_{\boldsymbol{U}, F}=\left\{x \in\left(\boldsymbol{G}^{l^{\prime} \sigma}\right)^{0} \mid x^{-1 F} x \in \boldsymbol{U}\right\}$.

Proof. Part (i) is an obvious computation and shows that if $x \in \boldsymbol{G}^{\sigma l}$ then $x^{\sigma^{F} \lambda^{-1}} \in \boldsymbol{G}^{l^{\prime} \sigma}$. To prove (ii), it remains to show that if $x \in \boldsymbol{G}^{\sigma l}$ then ${ }^{F}\left(x^{\sigma^{F} \lambda^{-1}}\right)=\left({ }^{\sigma F} x\right)^{\sigma^{F} \lambda^{-1}}$. From $x^{\sigma}=x^{l^{-1}}=x^{\lambda^{-1} \cdot F_{\lambda}}$, we get $x^{\sigma^{F} \lambda^{-1}}=x^{\lambda^{-1}}$, whence ${ }^{F}\left(x^{\sigma^{F} \lambda^{-1}}\right)=\left({ }^{F} x\right)^{F \lambda^{-1}}=$ $\left(\left({ }^{\sigma F} x\right)^{\sigma}\right)^{F \lambda^{-1}}=\left({ }^{\sigma F} x\right)^{\sigma^{F} \lambda^{-1}}$.

Applying Lemmas 8.2 and 8.3 we get

$\left({ }^{*} R_{\boldsymbol{L}^{1}}^{\boldsymbol{G}^{1}} \operatorname{sh}_{F / \sigma F}(\chi)\right)(\sigma l u)=$

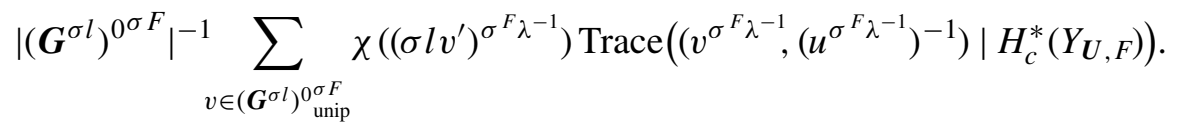


Lemma 8.4. Assume that the characteristic is good for $\boldsymbol{G}^{\sigma 0}$, where $\sigma$ is a quasicentral element of $\boldsymbol{G}$. Then it is also good for $\left(\boldsymbol{G}^{s}\right)^{0}$, where s is any quasi-semisimple element of $\boldsymbol{G}^{0} \cdot \sigma$.

Proof. Let $\Sigma_{\sigma}$ (resp. $\Sigma_{s}$ ) be the root system of $\boldsymbol{G}^{\sigma 0}\left(\right.$ resp. $\left.\left(\boldsymbol{G}^{s}\right)^{0}\right)$. By definition, a characteristic $p$ is good for a reductive group if for no closed subsystem of its root system the quotient of the generated lattices has $p$-torsion. The system $\Sigma_{s}$ is not a closed subsystem of $\Sigma_{\sigma}$ in general, but the relationship is expounded in [Digne and Michel 2002]: let $\Sigma$ be the root system of $\boldsymbol{G}^{0}$ with respect to a $\sigma$-stable pair $\boldsymbol{T} \subset \boldsymbol{B}$ of a maximal torus and a Borel subgroup of $\boldsymbol{G}^{0}$. Up to conjugacy, we may assume that $s$ also stabilizes that pair. Let $\bar{\Sigma}$ denote the set of sums of the $\sigma$-orbits in $\Sigma$, and $\Sigma^{\prime}$ the set of averages of the same orbits. Then $\Sigma^{\prime}$ is a nonnecessarily reduced root system, but $\Sigma_{\sigma}$ and $\Sigma_{s}$ are subsystems of $\Sigma^{\prime}$ and are reduced. The system $\bar{\Sigma}$ is reduced, and the set of sums of orbits whose average is in $\Sigma_{\sigma}$ (resp. $\left.\Sigma_{s}\right)$ is a closed subsystem that we denote by $\bar{\Sigma}_{\sigma}$ (resp. $\bar{\Sigma}_{s}$ ).

We now need a generalization of [Bourbaki 1981, chapitre VI, §1.1, lemme 1]:

Lemma 8.5. Let $\mathcal{L}$ be a finite set of lines generating a vector space $V$ over a field of characteristic 0 ; then two reflections of $V$ which stabilize $\mathcal{L}$ and have a common eigenvalue $\zeta \neq 1$ with $\zeta$-eigenspace the same line of $\mathcal{L}$ are equal.

Proof. Here we mean by reflection an element $s \in \mathrm{GL}(V)$ such that $\operatorname{ker}(s-1)$ is a hyperplane. Let $s$ and $s^{\prime}$ be reflections as in the statement. The product $s^{-1} s^{\prime}$ stabilizes $\mathcal{L}$, so it has a power which fixes $\mathcal{L}$, and thus is semisimple. On the other hand, $s^{-1} s^{\prime}$ by assumption fixes one line $L \in \mathcal{L}$ and induces the identity on $V / L$, and thus is unipotent. Being semisimple and unipotent, it has to be the identity.

It follows from Lemma 8.5 that two root systems with proportional roots have the same Weyl group, and thus the same good primes; therefore:

- $\Sigma_{s}$ and $\bar{\Sigma}_{s}$ have the same good primes, as well as $\Sigma_{\sigma}$ and $\bar{\Sigma}_{\sigma}$.

- The bad primes for $\bar{\Sigma}_{s}$ are a subset of those for $\bar{\Sigma}$, since it is a closed subsystem.

It only remains to show that the good primes for $\bar{\Sigma}$ are the same as for $\bar{\Sigma}_{\sigma}$, which can be checked case by case: we can reduce to the case where $\Sigma$ is irreducible, in which case these systems coincide except when $\Sigma$ is of type $A_{2 n}$; but in this case, $\bar{\Sigma}$ is of type $B_{n}$ and $\Sigma_{\sigma}$ is of type $B_{n}$ or $C_{n}$, which have the same set $\{2\}$ of bad primes.

Since the characteristic is good for $\boldsymbol{G}^{\sigma 0}$, hence also for $\left(\boldsymbol{G}^{\sigma l}\right)^{0}$ by Lemma 8.4, the elements $v^{\prime}$ and $v$ are conjugate in $\left(\boldsymbol{G}^{\sigma l}\right)^{0^{\sigma F}}$ (see [Digne and Michel 1985, IV, corollaire 1.2]). By Lemma 8.3(ii), the element $v^{\sigma^{F} \lambda^{-1}}$ runs over the unipotent elements of $\left(\boldsymbol{G}^{l^{\prime} \sigma}\right)^{0^{F}}$ when $v$ runs over $\left(\boldsymbol{G}^{\sigma l}\right)^{0^{\sigma F}}$ unip. Moreover, using the equality 
$\left|\left(\boldsymbol{G}^{\sigma l}\right)^{0^{\sigma F}}\right|=\left|\left(\boldsymbol{G}^{l^{\prime} \sigma}\right)^{0^{F}}\right|$, we get

(*)

$$
\begin{aligned}
& \left({ }^{*} R_{\boldsymbol{L}^{1}}^{\boldsymbol{G}^{1}} \operatorname{sh}_{F / \sigma F}(\chi)\right)(\sigma l u)= \\
& \quad \frac{1}{\left|\left(\boldsymbol{G}^{l^{\prime} \sigma}\right)^{0}{ }^{F}\right|} \sum_{u_{1} \in\left(\boldsymbol{G}^{l^{\prime} \sigma}\right)^{0^{F}} \text { unip }} \chi\left(u_{1} l^{\prime} \sigma\right) \operatorname{Trace}\left(\left(u_{1},\left(u^{\sigma^{F} \lambda^{-1}}\right)^{-1}\right) \mid H_{c}^{*}\left(Y_{\boldsymbol{U}, F}\right)\right) .
\end{aligned}
$$

On the other hand, by Lemma 8.2 applied with $v=u$, we have

$$
\begin{aligned}
\left(\operatorname{sh}_{F / \sigma F}{ }^{*} R_{\boldsymbol{L}^{1}}^{G^{1}}(\chi)\right)(\sigma l u) & ={ }^{*} R_{\boldsymbol{L}^{1}}^{G^{1}}(\chi)\left((\sigma l u)^{\sigma^{F} \lambda^{-1}}\right) \\
& ={ }^{*} R_{\boldsymbol{L}^{1}}^{\boldsymbol{G}^{1}}(\chi)\left(l^{\prime} \sigma \cdot u^{\sigma^{F} \lambda^{-1}}\right),
\end{aligned}
$$

where the second equality holds by Lemma 8.3(i). By the character formula this is equal to the right-hand side of formula $(*)$.

\section{References}

[Bourbaki 1981] N. Bourbaki, Éléments de mathématique: Groupes et algèbres de Lie, Chapitres 4, 5 et 6, Masson, Paris, 1981. MR 83g:17001 Zbl 0498.12001

[Digne 1999] F. Digne, "Descente de Shintani et restriction des scalaires”, J. London Math. Soc. (2) 59:3 (1999), 867-880. MR 2001a:20081 Zbl 0947.20025

[Digne and Michel 1985] F. Digne and J. Michel, Fonctions L des variétés de Deligne-Lusztig et descente de Shintani, Mém. Soc. Math. France (N.S.) 20, Société Mathématique de France, Paris, 1985. MR 87h:20071 Zbl 0608.20027

[Digne and Michel 1991] F. Digne and J. Michel, Representations of finite groups of Lie type, London Mathematical Society Student Texts 21, Cambridge Univ. Press, 1991. MR 92g:20063 Zbl 0815.20014

[Digne and Michel 2002] F. Digne and J. Michel, "Points fixes des automorphismes quasi-semisimples", C. R. Math. Acad. Sci. Paris 334:12 (2002), 1055-1060. MR 2003j:20081 Zbl 1001.20043

[DM 1994] F. Digne and J. Michel, "Groupes réductifs non connexes”, Ann. Sci. École Norm. Sup.

(4) 27:3 (1994), 345-406. MR 95f:20068 Zbl 0846.20040

[Eftekhari 1996] M. Eftekhari, "Faisceaux caractères sur les groupes non connexes", J. Algebra 184:2 (1996), 516-537. MR 98a:20047 Zbl 0870.20027

[Lawther et al. 2014] R. Lawther, M. W. Liebeck, and G. M. Seitz, "Outer unipotent classes in automorphism groups of simple algebraic groups", Proc. Lond. Math. Soc. (3) 109:3 (2014), 553595. MR 3260287 Zbl 06355609

[Lehrer 1992] G. I. Lehrer, "Rational tori, semisimple orbits and the topology of hyperplane complements”, Comment. Math. Helv. 67:2 (1992), 226-251. MR 93e:20065 Zbl 0816.20037

[Lusztig 2003] G. Lusztig, "Character sheaves on disconnected groups, I", Represent. Theory 7 (2003), 374-403. MR 2006d:20090a Zbl 1072.20052

[Lusztig 2004a] G. Lusztig, "Character sheaves on disconnected groups, II", Represent. Theory 8 (2004), 72-124. MR 2006d:20090b Zbl 1073.20036

[Lusztig 2004b] G. Lusztig, "Character sheaves on disconnected groups, III", Represent. Theory 8 (2004), 125-144. MR 2006d:20090c Zbl 1074.20031

[Lusztig 2004c] G. Lusztig, "Character sheaves on disconnected groups, IV", Represent. Theory 8 (2004), 145-178. MR 2006d:20090d Zbl 1075.20013 
[Lusztig 2004d] G. Lusztig, "Character sheaves on disconnected groups, V", Represent. Theory 8 (2004), 346-376. MR 2005h:20111 Zbl 1076.20031

[Lusztig 2004e] G. Lusztig, "Character sheaves on disconnected groups, VI", Represent. Theory 8 (2004), 377-413. MR 2005h:20112 Zbl 1077.20058

[Lusztig 2005] G. Lusztig, "Character sheaves on disconnected groups, VII", Represent. Theory 9 (2005), 209-266. MR 2006e:20089 Zbl 1078.20047

[Lusztig 2006a] G. Lusztig, "Character sheaves on disconnected groups, IX", Represent. Theory 10 (2006), 353-379. MR 2008e:20078 Zbl 1161.20039

[Lusztig 2006b] G. Lusztig, "Character sheaves on disconnected groups, VIII", Represent. Theory 10 (2006), 314-352. MR 2008f:20122 Zbl 1160.20038

[Lusztig 2009] G. Lusztig, "Character sheaves on disconnected groups, X”, Represent. Theory 13 (2009), 82-140. MR 2010i:20058 Zbl 1233.20041

[Steinberg 1968] R. Steinberg, Endomorphisms of linear algebraic groups, Mem. Amer. Math. Soc. 80, Amer. Math. Soc., Providence, R.I., 1968. MR 37 \#6288 Zbl 0164.02902

Received March 3, 2015. Revised March 5, 2015.

FRANÇOIS DIGNE

Laboratoire Amiénois de Mathématique Fondamentale et ApPliquée

Université de PiCARdie Jules Verne

CNRS UMR 7352

80039 AMIENS CEDEX

FRANCE

digne@u-picardie.fr

JEAN MICHEL

Institut de Mathématiques de Jussieu - PARis Rive GAuChe

UNIVERSITÉ DENIS DIDEROT

BÂTIMENT SOPHIE GERMAIN

75013 PARIS

FRANCE

jean.michel@imj-prg.fr 


\title{
PACIFIC JOURNAL OF MATHEMATICS
}

\author{
msp.org/pjm
}

Founded in 1951 by E. F. Beckenbach (1906-1982) and F. Wolf (1904-1989)

\section{EDITORS}

Don Blasius (Managing Editor)

Department of Mathematics

University of California

Los Angeles, CA 90095-1555

blasius@math.ucla.edu

\author{
Paul Balmer \\ Department of Mathematics \\ University of California \\ Los Angeles, CA 90095-1555 \\ balmer@math.ucla.edu \\ Robert Finn \\ Department of Mathematics \\ Stanford University \\ Stanford, CA 94305-2125 \\ finn@math.stanford.edu \\ Sorin Popa \\ Department of Mathematics \\ University of California \\ Los Angeles, CA 90095-1555 \\ popa@math.ucla.edu
}

\author{
Vyjayanthi Chari \\ Department of Mathematics \\ University of California \\ Riverside, CA 92521-0135 \\ chari@math.ucr.edu \\ Kefeng Liu \\ Department of Mathematics \\ University of California \\ Los Angeles, CA 90095-1555 \\ liu@math.ucla.edu \\ Jie Qing \\ Department of Mathematics \\ University of California \\ Santa Cruz, CA 95064 \\ qing@ cats.ucsc.edu
}

\section{PRODUCTION}

Silvio Levy, Scientific Editor, production@msp.org

\section{SUPPORTING INSTITUTIONS}

ACADEMIA SINICA, TAIPEI

CALIFORNIA INST. OF TECHNOLOGY

INST. DE MATEMÁTICA PURA E APLICADA

KEIO UNIVERSITY

MATH. SCIENCES RESEARCH INSTITUTE

NEW MEXICO STATE UNIV.

OREGON STATE UNIV.

\author{
STANFORD UNIVERSITY \\ UNIV. OF BRITISH COLUMBIA \\ UNIV. OF CALIFORNIA, BERKELEY \\ UNIV. OF CALIFORNIA, DAVIS \\ UNIV. OF CALIFORNIA, LOS ANGELES \\ UNIV. OF CALIFORNIA, RIVERSIDE \\ UNIV. OF CALIFORNIA, SAN DIEGO \\ UNIV. OF CALIF., SANTA BARBARA
}

\author{
Daryl Cooper \\ Department of Mathematics \\ University of California \\ Santa Barbara, CA 93106-3080 \\ cooper@math.ucsb.edu \\ Jiang-Hua Lu \\ Department of Mathematics \\ The University of Hong Kong \\ Pokfulam Rd., Hong Kong \\ jhlu@maths.hku.hk \\ Paul Yang \\ Department of Mathematics \\ Princeton University \\ Princeton NJ 08544-1000 \\ yang@math.princeton.edu
}

These supporting institutions contribute to the cost of publication of this Journal, but they are not owners or publishers and have no responsibility for its contents or policies.

See inside back cover or msp.org/pjm for submission instructions.

The subscription price for 2015 is US \$420/year for the electronic version, and \$570/year for print and electronic.

Subscriptions, requests for back issues and changes of subscribers address should be sent to Pacific Journal of Mathematics, P.O. Box 4163, Berkeley, CA 94704-0163, U.S.A. The Pacific Journal of Mathematics is indexed by Mathematical Reviews, Zentralblatt MATH, PASCAL CNRS Index, Referativnyi Zhurnal, Current Mathematical Publications and Web of Knowledge (Science Citation Index).

The Pacific Journal of Mathematics (ISSN 0030-8730) at the University of California, c/o Department of Mathematics, 798 Evans Hall \#3840, Berkeley, CA 94720-3840, is published twelve times a year. Periodical rate postage paid at Berkeley, CA 94704, and additional mailing offices. POSTMASTER: send address changes to Pacific Journal of Mathematics, P.O. Box 4163, Berkeley, CA 94704-0163.

PJM peer review and production are managed by EditFLOW ${ }^{\circledR}$ from Mathematical Sciences Publishers.

\section{PUBLISHED BY}

\section{mathematical sciences publishers \\ nonprofit scientific publishing}

http://msp.org/

(C) 2015 Mathematical Sciences Publishers 


\title{
PACIFIC JOURNAL OF MATHEMATICS
}

\author{
Volume 279 No. 1-2 December 2015
}

In memoriam: Robert Steinberg

Robert Steinberg (1922-2014): In memoriam V. S. VARADARAJAN

Cellularity of certain quantum endomorphism algebras

HENNING H. ANDERSEN, GUSTAV I. LEHRER and RUIBIN ZHANG

Lower bounds for essential dimensions in characteristic 2 via orthogonal representations ANTONIO BABIC and VLADIMIR CHERNOUSOV

Cocharacter-closure and spherical buildings

Michael Bate, Sebastian Herpel, Benjamin Martin and Gerhard RöHrLe

Embedding functor for classical groups and Brauer-Manin obstruction

Eva Bayer-Fluckiger, Ting-Yu LeE and Raman Parimala

On maximal tori of algebraic groups of type $G_{2}$

Constantin Beli, PhilipPe Gille and Ting-Yu LeE

On extensions of algebraic groups with finite quotient

MICHEL BRION

Essential dimension and error-correcting codes

SHANE CERNELE and ZiNOVy REICHSTEIN

Notes on the structure constants of Hecke algebras of induced representations of finite Chevalley groups

Charles W. CuRTis

Complements on disconnected reductive groups

FRANÇOIS DIGNE and JEAN MICHEL

Extending Hecke endomorphism algebras

Jie Du, Brian J. Parshall and LeOnard L. SCOTT

Products of partial normal subgroups

ELLEN HENKE

Lusztig induction and $\ell$-blocks of finite reductive groups

RADHA KESSAR and GUNTER MALLE

Free resolutions of some Schubert singularities

Manoj Kummini, Venkatramani Lakshmibai, Pramathanath Sastry and C. S. Seshadri

Free resolutions of some Schubert singularities in the Lagrangian Grassmannian

VenKatramani LAKSHMibai and ReUVEN HODGES

Distinguished unipotent elements and multiplicity-free subgroups of simple algebraic groups

Martin W. Liebeck, Gary M. Seitz and Donna M. Testerman

Action of longest element on a Hecke algebra cell module

GEORGE LUSZTIG

Generic stabilisers for actions of reductive groups

BENJAMIN MARTIN

On the equations defining affine algebraic groups

VLADIMIR L. POPOV

Smooth representations and Hecke modules in characteristic $p$

PETER SCHNEIDER

On CRDAHA and finite general linear and unitary groups

BHAMA SRINIVASAN

Weil representations of finite general linear groups and finite special linear groups PHAM HUU TIEP

The pro- $p$ Iwahori Hecke algebra of a reductive $p$-adic group, $\mathrm{V}$ (parabolic induction) MARIE-FRANCE VIGNÉRAS

Acknowledgement 